\title{
Herschel-PACS observations of far-IR lines in young stellar objects
}

\section{I. [OI] and $\mathrm{H}_{2} \mathrm{O}$ at $63 \mu \mathrm{m}^{\star, \star \star}$}

\author{
P. Riviere-Marichalar ${ }^{1}$, B. Merín ${ }^{1}$, I. Kamp ${ }^{2}$, C. Eiroa $^{3}$, and B. Montesinos ${ }^{4}$ \\ 1 European Space Astronomy Centre (ESA), PO Box 78, 28691 Villanueva de la Cañada, Spain \\ e-mail: priviere@sciops.esa.int \\ 2 Kapteyn Astronomical Institute, University of Groningen, PO Box 800, 9700 AV Groningen, The Netherlands \\ 3 Dep. de Física Teórica, Fac. de Ciencias, UAM Campus Cantoblanco, 28049 Madrid, Spain \\ 4 Depto. Astrofísica, Centro de Astrobiología (CAB, INTA-CSIC), PO Box 78, ESAC Campus, 28691 Villanueva de la Cañada, \\ Madrid, Spain
}

Received 25 November 2015 / Accepted 28 July 2016

\begin{abstract}
Context. Gas plays a major role in the dynamical evolution of young stellar objects (YSOs). Its interaction with the dust is the key to our understanding planet formation later on in the protoplanetary disc stage. Studying the gas content is therefore a crucial step towards understanding YSO and planet formation. Such a study can be made through spectroscopic observations of emission lines in the far-infrared, where some of the most important gas coolants emit, such as the [OI] ${ }^{3} \mathrm{P}_{1} \rightarrow{ }^{3} \mathrm{P}_{2}$ transition at $63.18 \mu \mathrm{m}$.

Aims. We provide a compilation of observations of far-IR lines in 362 YSOs covering all evolutionary stages, from Class 0 to Class III with debris discs. In the present paper we focus on [OI] and o- $\mathrm{H}_{2} \mathrm{O}$ emission at $63 \mu \mathrm{m}$.

Methods. We retrieved all the available Herschel-PACS spectroscopic observations at $63 \mu \mathrm{m}$ that used the dominant observing mode, the chop-nod technique. We provide measurements of line fluxes for the [OI] ${ }^{3} \mathrm{P}_{1} \rightarrow{ }^{3} \mathrm{P}_{2}$ and o- $\mathrm{H}_{2} \mathrm{O} 8_{08} \rightarrow 717$ transitions at $63 \mu \mathrm{m}$ computed using different methods. Taking advantage of the PACS IFU, we checked for spatially extended emission and also studied multiple dynamical components in line emission.

Results. The final compilation consists of line and continuum fluxes at $63 \mu \mathrm{m}$ for a total of 362 young stellar objects (YSOs). We detect [OI] line emission at $63 \mu \mathrm{m}$ in 194 sources out of 362, and line absorption in another five sources. o- $\mathrm{H}_{2} \mathrm{O}$ was detected in 42 sources. We find evidence of extended [OI] emission in 77 sources, and detect $3 \sigma$ residual emission in 71 of them. The number of sources showing extended emission decays from Class 0 to Class II. We also searched for different components contributing to the line emission, and found evidence for multiple components in 30 sources. We explored correlations between line emission and continuum emission and found a clear correlation between WISE fluxes from 4.6 to $22 \mu \mathrm{m}$ and [OI] line emission. We conclude that the observed emission is typically a combination of disc, envelope and jet emission.
\end{abstract}

Key words. astrochemistry - circumstellar matter - stars: evolution - stars: protostars

\section{Introduction}

Young stellar objects (YSO) are complex sources consisting of many components, such as the central source (protostellar or stellar), an envelope made of gas and dust, a circumstellar disc, stellar and disc winds, and large-scale collimated jets. Each of the components can contribute to different observables, such as photometry and line fluxes. A detailed study is therefore needed to elucidate the contribution of each component.

In the initial stages of stellar formation, Class 0 and I protostars (Lada \& Wilking 1984; Lada 1987; Andre et al. 1993) are surrounded by an envelope. Discs are clearly detected around Class I sources. Class I sources later evolve to Class II sources, in which the central star is already formed and the envelope dispersed. The formation of a dust opacity hole in the inner disc leads to the formation of the so-called transitional discs

\footnotetext{
* The catalogue of observations and fluxes (Tables B.1-B.3) is only available at the CDS via anonymous ftp to cdsarc.u-strasbg.fr $(130.79 .128 .5)$ or via

http://cdsarc.u-strasbg.fr/viz-bin/qcat?J/A+A/594/A59 $\star \star$ Herschel is an ESA space observatory with science instruments provided by European-led Principal Investigator consortia and with important participation from NASA.
}

(Strom et al. 1989). Many mechanisms have been used to explain the formation of the inner opacity holes, including planet formation. At $10 \mathrm{Myr}$, most primordial discs have been dispersed (Strom et al. 1989), but destructive collisions between planetesimals can repopulate the circumstellar environment with dust, resulting in the so-called debris discs.

Young stellar objects can also be classified according to their masses. The so-called T Tauri stars are variable stars showing bright emission lines with stellar masses $M_{*}<2.0 M_{\odot}$, while HAeBe stars are the high-mass counterparts of $\mathrm{T}$ Tauri stars $\left(2.0<M / M_{\odot}<8.0\right)$.

Although gas is thought to dominate the mass budget during the primordial stages (Class 0 to II), little is known about its mass and spatial distribution, mostly because it is difficult to detect $\mathrm{H}_{2}$, which lacks a permanent dipole moment. However, to learn about the formation of planets, we need to understand the chemical evolution of gas and dust.

The Herschel Space Observatory (Pilbratt et al. 2010) produced thousands of observations of YSOs during its four-year mission. The most widely used instrument was the Photodetector Array Camera and Spectrometer (PACS, Poglitsch et al. 2010), which can spectroscopically observe the far-IR 50-250 $\mu \mathrm{m}$ 
range. Furthermore, it also performed photometric observations at 70, 100 and $160 \mu \mathrm{m}$ with great sensitivity. One of the most interesting characteristics of the PACS spectrometer is its Integral Field Unit (IFU), divided into 25 spaxels distributed in a regular grid covering $47^{\prime \prime} \times 47^{\prime \prime}$. The IFU allows us to study the spatial distribution of the continuum and line emission.

Some studies have surveyed [OI], $\mathrm{CO}, \mathrm{OH}$, and $\mathrm{H}_{2} \mathrm{O}$ emission in objects belonging to different stellar associations and moving groups using Herschel (Donaldson et al. 2012; Howard et al. 2013; Green et al. 2013; Mathews et al. 2013; Lindberg et al. 2014; Riviere-Marichalar et al. 2013, 2014, 2015). Other studies have focused on the analysis of individual sources (Meeus et al. 2010; van Kempen et al. 2010b,a; Sturm et al 2010; Thi et al. 2010; Tilling et al. 2012; Lebreton et al. 2012; Riviere-Marichalar et al. 2012a; Thi et al. 2013). However, the spatial extension of the emission was discussed in only a few cases (Karska et al. 2013, 2014b; Nisini et al. 2013, 2015). The most extensively studied wavelength range is $63.0-63.4 \mu \mathrm{m}$, which includes two transitions, [OI ${ }^{3} \mathrm{P}_{1} \rightarrow{ }^{3} \mathrm{P}_{2}$ at $63.185 \mu \mathrm{m}$ and $\mathrm{o}-\mathrm{H}_{2} \mathrm{O} 8_{08} \rightarrow 7_{17}$ at $63.325 \mu \mathrm{m}$. [OI] emission has been detected in YSOs at all evolutionary stages, from Class 0 and I (Green et al. 2013) to Class II and transitional (Howard et al. 2013) and debris discs (Riviere-Marichalar et al. 2012b). o- $\mathrm{H}_{2} \mathrm{O}$ emission was observed around Class 0, I, II and transition discs, but not around debris discs.

Understanding the spatial distribution of far-IR lines emission is crucial, since it has been shown that envelopes, protoplanetary discs, and outflows can contribute to [OI] emission (van Kempen et al. 2010b; Podio et al. 2012; Karska et al. 2013). [OI] extended emission along the jet direction has been commonly observed, while molecular extended emission is observed in only a few cases (van Kempen et al. 2010b; Herczeg et al. 2012). Podio et al. (2012) and Karska et al. (2013) explained the extended emission as being produced by $\mathrm{J}$ - and C-shocks along the jet, and noted a decay in far-IR lines intensity from Class 0/I to Class II. Howard et al. (2013) studied a sample of Class II sources in Taurus, including sources with and without a jet or an outflow. The authors found a tight correlation between continuum emission at $63 \mu \mathrm{m}$ and [OI] emission, suggesting a disc origin for the line. However, sources with jets show a brighter [OI] emission for the same level of continuum, indicating a contribution from the jet. The authors did not find a correlation between disc mass (derived from sub-millimeter continuum emission) and $[\mathrm{OI}]$ line intensity, indicating that either the line is optically thick or it is a poor tracer of gas mass. Green et al. (2013) studied a sample of 30 embedded sources (Class 0 and I) from the DIGIT program (see e.g. van Kempen et al. 2010a; Sturm et al. 2010) and found a tight correlation between line intensity and $\mathrm{L}_{\mathrm{bol}}$.

In this paper, we present a compilation of 432 PACS spectroscopic observations of 362 YSOs and main-sequence stars with debris discs. We focus on the small wavelength range between 63.0 and $63.4 \mu \mathrm{m}$, which includes the [OI] transition at $63.185 \mu \mathrm{m}$ and the $\mathrm{o}-\mathrm{H}_{2} \mathrm{O}$ transition at $63.325 \mu \mathrm{m}$. Our wavelength range selection is motivated by the fact that the [OI] transition at $63.185 \mu \mathrm{m}$ typically is the strongest line coolant in protoplanetary discs (Gorti \& Hollenbach 2008). We leave the study of other transitions observed in PACS range mode for a future paper.

\section{Sample and observations}

The data were collected from fourteen different programs (see Table 1). The sample consists of 362 YSOs and main-sequence
Table 1. Overview of the programs used in this study.

\begin{tabular}{lll}
\hline \hline Program ID & Observations & $\begin{array}{l}\text { Sensitivity } \\
\left(10^{-18} \mathrm{~W} / \mathrm{m}^{2}\right)\end{array}$ \\
\hline GT1_vgeers_1 & - & 2.5 \\
KPOT_bdent_1 & 185 & 3.1 \\
KPOT_nevans_1 & 65 & 7.3 \\
KPGT_golofs01_1 & 6 & 2.9 \\
KPGT_evandish_1 & 28 & 8.6 \\
OT1_aschol_11 & 1 & 1.2 \\
OT1_cespaill_2 & 38 & 20.0 \\
OT1_ckiss_1 & 9 & 3.5 \\
OT1_gmeeus_1 & 2 & 28.0 \\
OT1_ipascucc_1 & 30 & 1.6 \\
OT1_maudar01_1 & 11 & 23.0 \\
OT1_vgeers_2 & 4 & 2.5 \\
OT2_amoor_3 & 2 & 3.8 \\
OT2_evandish_4 & 49 & 7.7 \\
\hline
\end{tabular}

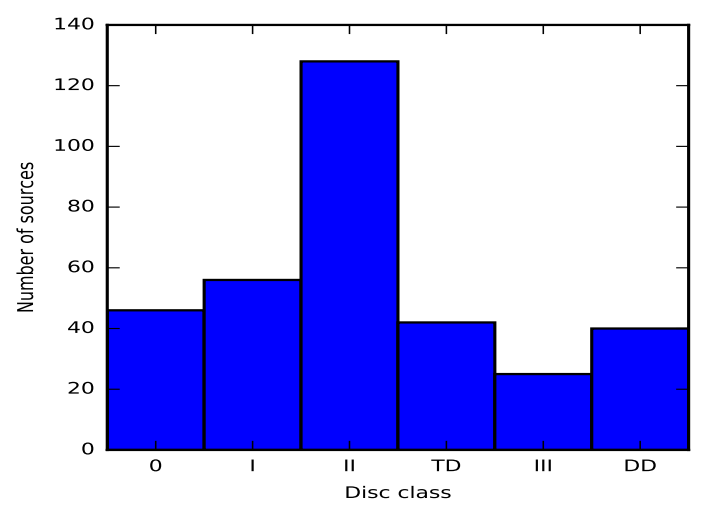

Fig. 1. Distribution of evolutionary stages in the observed sample.

stars with debris discs observed with PACS spectroscopy at $63 \mu \mathrm{m}$. The total number of observations was 432: 51 sources were observed twice, and another nine sources were observed three times. We only included pointed observations from programs that used the chop-nod technique to remove the telescope and background contribution. The sample includes objects in all the different evolutionary stages for circumstellar material, from Class 0 to Class III stars and to those that are later surrounded by debris discs (see Fig. 1). Highly embedded sources from the DIGIT program (Sturm et al. 2010) were included, together with highly embedded and high-mass protostellar envelopes from the WISH program (van Dishoeck et al. 2011). Transition discs are a particular case of Class II discs that have opened a gap in the inner part of the disc.

Throughout the paper we treat transition discs separately because of their importance in testing planet formation theories. When we refer to Class II discs, we therefore refer to full discs without an inner gap, in contrast to transition discs which show inner opacity holes. Because of the limited size of the samples and because of the similarities we found, we treat Class 0 and I sources in some sections as a single group of protostellar objects (see Sect. 4). Most of the stars in the sample belong to different star-forming regions and stellar associations, with ages in the range 1-40 Myr, including Tau, Cha, Cha II, Lupus, Lupus III, $\eta$ Cha, Upper Scorpius, TWA, BPMG, Tuc Hor, CrA, Serpens, Per, and Oph. The source names, positions, spectral type (for Class II and III sources), evolutionary status, and associations for YSOs in the sample are given in Table B.1. 
P. Riviere-Marichalar et al.: Herschel-PACS observations of far-IR lines in young stellar objects. I.

Observations were performed in two different modes depending on the program: line spectroscopy, covering 63.0 to $63.4 \mu \mathrm{m}$ (301 observations), and range spectroscopy (131 observations), covering 55 to $72 \mu \mathrm{m}$. Line spectroscopy observes a narrow spectral region centred on a certain spectroscopic transition ([OI] ${ }^{3} \mathrm{P}_{1} \rightarrow{ }^{3} \mathrm{P}_{2}$ in our case), and guarantees the detection of the full line profile for an unresolved line, with enough continuum coverage at either side of the line to allow for continuum measurements. Range spectroscopy covers a region around the lines of interest defined by the observer. The 63.0-63.4 $\mu \mathrm{m}$ range contains the [OI] ${ }^{3} \mathrm{P}_{1} \rightarrow{ }^{3} \mathrm{P}_{2}$ transition at $63.185 \mu \mathrm{m}$ and the o$\mathrm{H}_{2} \mathrm{O} 8_{18} \rightarrow 7_{07}$ transition at $63.325 \mu \mathrm{m}$. We focus in this paper on the study of the two lines in the 63 to $63.4 \mu \mathrm{m}$ wavelength range. Exposure times ranged from $851 \mathrm{~s}$ to $16420 \mathrm{~s}$, with $75 \%$ of the observations having $t_{\exp }<4000 \mathrm{~s}$. All the observations were performed in chop-nod mode, with a small chopper throw (1.5) for 299 sources $(\sim 71 \%)$, medium chopper throw $\left(3^{\prime}\right)$ for $13(\sim 3 \%)$ and large chopper throw $\left(6^{\prime}\right)$ for $107(\sim 26 \%)$. Most observations used only one nod cycle $(\sim 67 \%), \sim 26 \%$ used two nod cycles, four observations $(\sim 1 \%)$ used three nod cycles, $23(\sim 5 \%)$ used four nod cycles and six used five nod cycles $(1 \%)$.

\section{Data reduction}

The data were reduced using HIPE 12.0. The reduction was performed as follows. First, the observations were corrected for satellite movements. Then, saturated frames and frames with glitches were flagged and masked, and the chop-off position was subtracted from the chop-on position to remove the sky and telescope contribution. Then, the cubes were divided by the spectral response function, and after that, flat-fielding, using a straight line, was applied to improve the signal-to-noise ratio $(\mathrm{S} / \mathrm{N})$ of the continuum. We used a straight line for flatfielding. Then, the spectra were rebinned, with oversample $=2$ and upsample $=4$. Observations from the OT1_cespaill_2 and OT1_maudar01_1 programs, and some observations from the program KPOT_nevans_1, required oversample $=2$ and upsample $=2$ (native resolution of the instrument). Finally, the mean value from the two nod positions was computed.

Given the noise increment in the edges of the spectra, we only considered the spectral range from 63.0 to $63.4 \mu \mathrm{m}$ for line spectra and the range $62.5-63.9 \mu \mathrm{m}$ for range spectra. We subtracted the continuum contribution by fitting a first-order polynomial after masking a $\pm 3 \sigma$ (where $\sigma$ is the width of an unresolved line at the wavelength of interest) region around each transition present ([OI] and o- $\mathrm{H}_{2} \mathrm{O}$ at 63.185 and $63.325 \mu \mathrm{m}$, respectively). Line fluxes were computed by integrating a Gaussian fit to continuum-subtracted spectra of the spaxel with the highest flux. When the continuum was not detected to the $3 \sigma$ level, we extracted the spectrum from the central spaxel $(x=2, y=2)$. The spectrum from this spaxel was then aperture corrected. For some sources, a mis-pointing of Herschel causes the source to lie in between many spaxels, and therefore the computed flux is a lower limit to the actual flux. Adding the $3 \times 3$ spaxels around the position of the source gives a more accurate flux for these sources. The uncertainties on the line fluxes were computed as the integral of a Gaussian with a width equal to the fitted value and a peak equal to the noise of the continuum. Three-sigma upper limits were computed in a similar way as three times the integral of a Gaussian with a width equal to the instrumental value and a peak equal to the noise of the continuum. Given the spread in observing times between the different programs, the sensitivity limits are in the range $1.2 \times 10^{-18}$ to $2.8 \times 10^{-17} \mathrm{~W} / \mathrm{m}^{2}$ (see Table 1). We also obtained continuum fluxes at $63 \mu \mathrm{m}$ by computing the mean value of the baseline after excluding the $3 \sigma$ regions around the position of detectable lines, with errors being the standard deviation inside the same region.

\section{Results}

Examples of continuum-subtracted spectra for different evolutionary stages are shown in Fig. 2. We also show the Gaussian fits to the observed profiles used to compute line fluxes, together with residual plots at the bottom of each spectrum. The shift in the observed line centres compared to the theoretical ones in some sources might be due to mis-pointing of the telescope, since it is a known PACS effect that telescope mis-pointing results in a shift in wavelengths, which hinders concluding wether the line shift is real. The strength of the [OI] line compared to the $\mathrm{o}-\mathrm{H}_{2} \mathrm{O}$ is evident from the plots, with typical line ratios $\mathrm{F}_{[\mathrm{OI}]} / \mathrm{F}_{\mathrm{H}_{2} \mathrm{O}}$ in the range 2.4 to 29 . The only exception is BP Tau, where the o- $\mathrm{H}_{2} \mathrm{O}$ and [OI] line fluxes at $63 \mu \mathrm{m}$ are similar, within the errors.

\subsection{Line emission}

We detected the $[\mathrm{OI}]$ emission line at $63.185 \mu \mathrm{m}(3 \sigma)$ in 194 sources out of 362 observed $(0.54 \pm 0.04$ detection fraction). Line fluxes from the central spaxel are given in Table B.2. The detection fractions strongly depend on the evolutionary stage. Class 0 and I sources show very similar detection fractions $\left(0.87_{-0.07}^{+0.04}\right.$ and $0.93_{-0.06}^{+0.02}$, respectively), but show strong differences with Class II stars $\left(0.53_{-0.05}^{+0.06}\right.$, including both T Tauri and $\mathrm{HAeBe}$ stars). The detection fraction, also seems to depend on the stellar mass, since there is a strong difference between $\mathrm{T}$ Tauri and HAeBe stars $\left(0.42_{-0.05}^{+0.05}\right.$ and $0.96_{-0.08}^{+0.01}$, respectively). Transition discs and full Class II disc sources show detection fractions that are almost compatible $\left(0.56_{-0.08}^{+0.07}\right.$ for transition discs). Finally, [OI] was detected towards four debris disc sources (HD 172555, $\beta$ Pictoris, RXJ 18523-3700 and HD 141569), leading to the smallest detection fraction $\left(0.10_{-0.03}^{+0.07}\right)$. The detection fraction is likely to be even smaller, since HD 141569 is considered either a HAeBe star (Mendigutía et al. 2011) or a debris disc (Marsh et al. 2002).

$\mathrm{o}-\mathrm{H}_{2} \mathrm{O}$ at $63.325 \mu \mathrm{m}$ was detected in 43 out of 362 sources $\left(0.12 \pm 0.02\right.$ detection fraction). $\mathrm{o}^{-} \mathrm{H}_{2} \mathrm{O}$ line emission was only detected in sources where we also detected [OI] emission. Eight of these are Class 0 sources, two are intermediate Class 0/I sources, 20 are Class I sources, one (T Tau) is an intermediate Class I/II source, ten are Class II sources (eight of them are $\mathrm{T}$ Tauri stars, and two are HAeBe stars), and two are transitional discs.

[OI] line fluxes extracted from the central spaxel range from $4 \times 10^{-18}$ to $4 \times 10^{-14} \mathrm{~W} / \mathrm{m}^{2}$ and $\mathrm{o}-\mathrm{H}_{2} \mathrm{O}$ line fluxes range from $6 \times 10^{-18}$ to $7 \times 10^{-16} \mathrm{~W} / \mathrm{m}^{2}$. We show in Fig. 3 the distribution of [OI] (top) and $\mathrm{H}_{2} \mathrm{O}$ (bottom) luminosities for the different types of sources. Class 0 and I sources show a similar distribution of $[\mathrm{OI}]$ luminosities. However, they show quite different $\mathrm{H}_{2} \mathrm{O}$ distributions. Class II and transitional discs show more differences in their [OI] luminosity distributions than they do in their $\mathrm{H}_{2} \mathrm{O}$ luminosity distributions. The low number of $\mathrm{H}_{2} \mathrm{O}$ detections precludes any further comparison of the distributions. The vertical dashed lines in Fig. 3 show the median [OI] line luminosity for each class. We observe a clear evolutionary trend, with [OI] line luminosities that decrease from Class 0 to Class II and transitional, in agreement with the finding by 

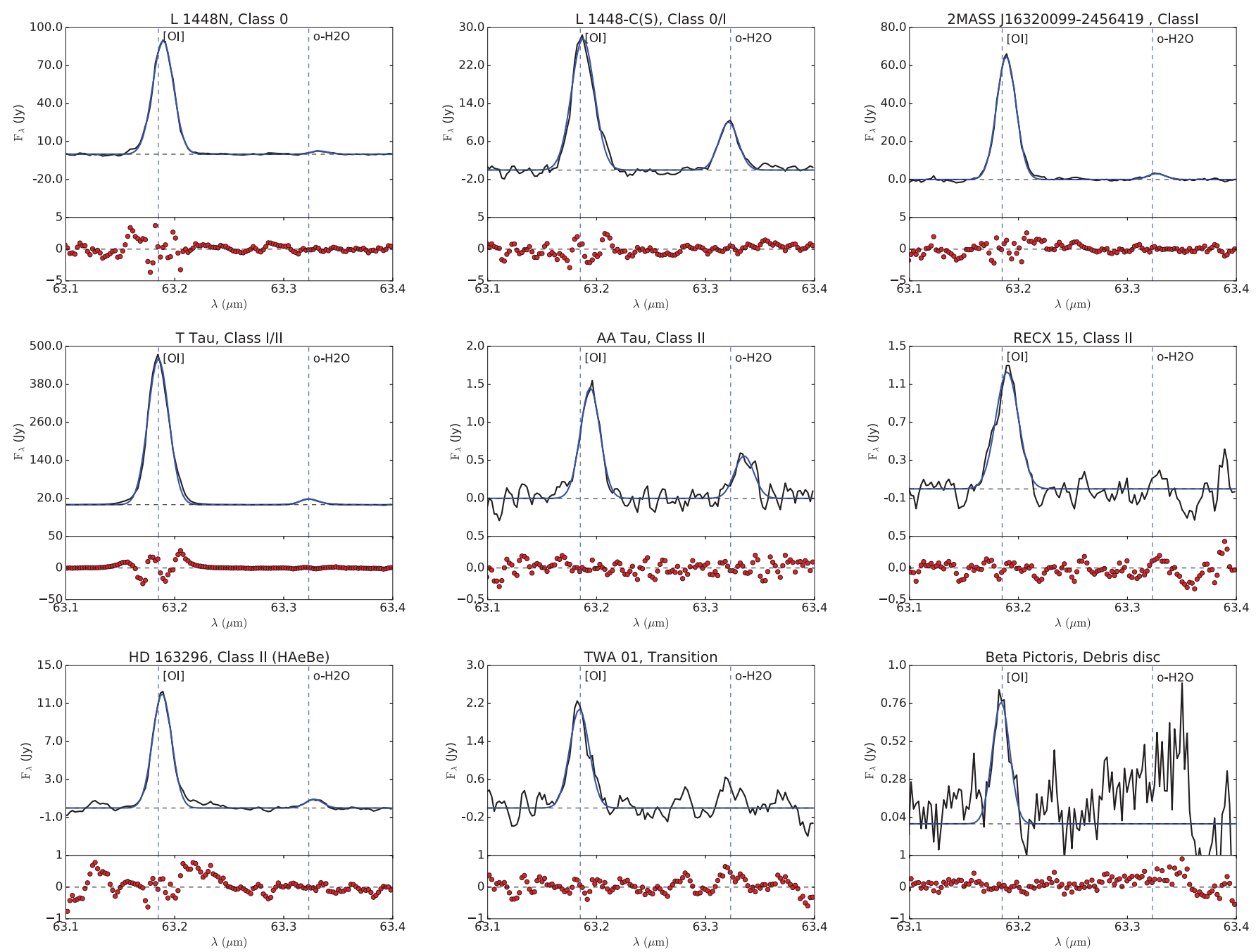

Fig. 2. Example of continuum-subtracted spectra of stars at different evolutionary stages. The black line shows the observed spectra, while the blue line shows a Gaussian fit. We show the rest-frame wavelength of the $[\mathrm{OI}]$ and $\mathrm{o}-\mathrm{H}_{2} \mathrm{O}$ emission lines and vertical dashed blue lines with labels identifying each line. Plots with the model residuals are shown at the bottom of each spectrum.

Podio et al. (2012) and Karska et al. (2013), where a decrease in molecular luminosity and total line luminosity was observed.

To test whether the differences of [OI] luminosity distributions between the different classes are real, we performed twodistribution Kolmogorov-Smirnov tests for the different pairs of datasets. Class 0 and I sources do not differ in their distribution of $[\mathrm{OI}]$ fluxes $(P=0.93)$. As we find no difference between both distributions, we combined the two distributions for an additional comparison with the other classes. The comparison with the distributions for Class II bear strong differences: $P \ll 10^{-3}$ for Class II sources compared to Class 0 and I sources, where Class II includes full disc T Tauris, transition discs T Tauris and HAeBe stars. The difference is dominated by low-mass stars, since the probability is $P \ll 10^{-3}$ when HAeBe stars are excluded from the comparison. The comparison between T Tauri stars and HAeBe stars also shows strong differences $\left(P \sim 10^{-3}\right)$. We furthermore compared the distribution of luminosities for $\mathrm{T}$ Tauri stars surrounded by full discs and those surrounded by transitional discs, and found a probability $P \sim 10^{-3}$ that both populations are build from the same distribution. We did not perform the comparison for debris discs sources due to the small number of detections. However, we highlight the large dispersion of [OI] fluxes observed in debris discs, with only four detections covering more than two orders of magnitude. Figure 3 also shows an evolution of the typical [OI] luminosity with class, with the peak of the distribution having its maximum at fainter luminosities when moving from Class 0 to Class II and transitional discs.

It is important to note that many sources suffer from mispointing, affecting both the fluxes and the future assessment of extended emission (Sect. 4.4). This mis-pointing can be in the form of small shifts, which makes the flux from the central spaxel a poor estimate of the real value. In other cases, the shift can cause the source to be located in a spaxel other than the central one. In these cases, the sources can again be properly centred inside this spaxel or it can be shifted. These sources are marked in Table B. 2 with an asterisk.

\subsection{Line absorption}

Five sources in the sample showed line absorption, at least in the central spaxel. These sources are AFGL 2591, G327-0.6, G34.26+0.15, NGC 6334-I, and W33A. Their spectra are shown in Fig. 5. They are part of the WISH sample, and are classified as high-mass YSOs (van Dishoeck et al. 2011). Since the observations were performed using the chop-nod technique, we 

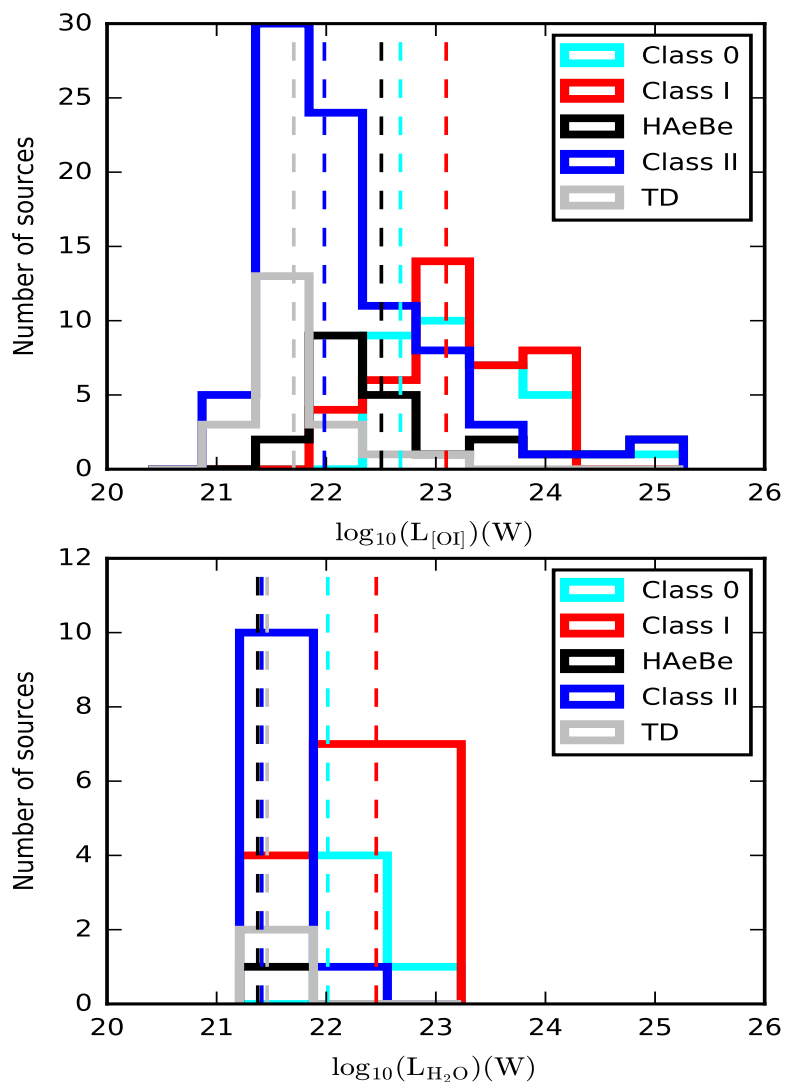

Fig. 3. Top: histogram for [OI] line luminosities in the observed sample. Bottom: histogram for $\mathrm{H}_{2} \mathrm{O}$ line luminosities in the observed sample. The vertical dashed lines show the median luminosity for each class.

inspected the on and off positions separately. We always found absorption in the on position, and only in one case, W33A, we observe faint emission in the off position. Therefore, we conclude that the observed absorption features are real.

The sources showing absorption were discussed in detail by Karska et al. (2014a). For two sources, namely AFGL 2591 and NGC 6334-I, the profile features the shape of a P-Cygni profile (Karska et al. 2014a). Line maps for sources showing absorption in the [OI] transition at $63.185 \mu \mathrm{m}$ are shown in Fig. 6, where we can see that absorption peaks at the positions were the continuum reaches the maximum. Absorption towards NGC 6334-V was previously reported by Kraemer et al. (1998), who attributed the absorption to cooler or less dense gas in the foreground core cloud.

Even if all the sources showing line absorption were highmass YSOs, we cannot conclude that high-mass envelopes lead to absorption, since another three high-mass YSOs in the sample, namely DR $21(\mathrm{OH})$, NGC 7538 IRS1, and W3-IRS5 show prominent emission. None of the eight sources showed $\mathrm{o}-\mathrm{H}_{2} \mathrm{O}$ emission or absorption at $63.323 \mu \mathrm{m}$.

\subsection{Continuum and line variability}

A total of 60 sources were observed multiple times (see Sect. 2, the discussed fluxes are shown in Table B.2). We can use this subsample to gain insight on line and continuum variability in the far-IR. In terms of line emission, we can distinguish three groups:

- Sources that show fluxes that agree, within the uncertainties. This group contains 38 sources.
- Sources that show fluxes that do not match becuase of a mis-pointing in one (or all) the observations. This groups contains 16 sources. Mis-pointed sources mostly come from the GASPS program, where a problem with the pointing of Taurus sources has been highlighted by Howard et al. (2013). These sources are properly flagged in Table B.2.

- Sources with fluxes that do not match, and where the disagreement is not due to mis-pointing, but most likely to real variability or unknown instrumental effects. This group includes six sources: HD 100453, HD 139614, HD 142527, HD 36112, IRAS 04016+2610, and SAO 206462. The spectra obtained at different epochs for these sources are shown in Fig. 7. Five of these (HD 100453, HD 139614, HD 142527, HD 36112 and SAO 206462) are HAeBe stars, and one (IRAS 04016+2610) is a Class 0/I embedded source. For IRAS $04016+2610$ the difference in the fluxes could be due to the different observational techniques used, since one of the observations is a line spectrum, while the other is a range spectrum. Furthermore, all sources are variable at most at the $3 \sigma$ level. For HD 100453, three observations are available, and none of them shows compatible line fluxes at the $1 \sigma$ level, but they are compatible at the $3 \sigma$ level. Furthermore, the continuum sources are not compatible at either the 1 or $3 \sigma$ levels.

Follow-up of [OI] line emission at $63 \mu \mathrm{m}$ is needed to understand line variability and rule out instrumental effects.

\subsection{Extended emission}

The Integral Field Unit (IFU) used for PACS spectroscopy allowed us to study whether the emission is extended or not. We first compared the flux derived from the central spaxel (or the spaxel with the highest signal) to those derived from co-adding the nine central spaxels (test 1) and the 25 spaxels (test 2). Finally, we also compared the flux from the central nine spaxels with that of the 25 spaxels (test 3 ). If the emission is extended, we expect the co-added fluxes to be substantially higher than the flux from the central spaxel. This stronger emission from co-added spaxels can be due to extended emission or to the presence of multiple sources. On the one hand, if the flux from the 25 spaxels were higher than the flux from the central spaxel, but the flux from the central were coincident with the flux from the central spaxel, then the most likely explanation would be the presence of another source (or sources) in the outer spaxels. On the other hand, if we were to detect higher fluxes only when the central nine spaxels are considered, then the difference would most likely be due to extended emission. We are aware that by co-adding the different spaxels, the signal detected in one of them can be diluted when the other spaxels are noisedominated.

We show in Table B.3 the computed fluxes for sources detected with at least one of the two methods. In Fig. 4, we compare the different fluxes computed to test for extended emission. When the difference in flux is larger than three times the quadratic sum of the uncertainties, we consider that the emission is extended. Sources with extended emission are shown as red dots in Fig. 4. When test 1 was used, 69 sources showed extended emission, 59 of which belonged to Class 0 and I, 9 belonging to Class II and transitional (including both T Tauri and HAeBe stars) and one is a highly embedded source with unknown class (RCrA-IRS7A). When test 2 was used, 69 sources showed extended emission, 56 of which belonged to Class 0 and I, 12 to Class II and transitional, and one is a highly embedded 

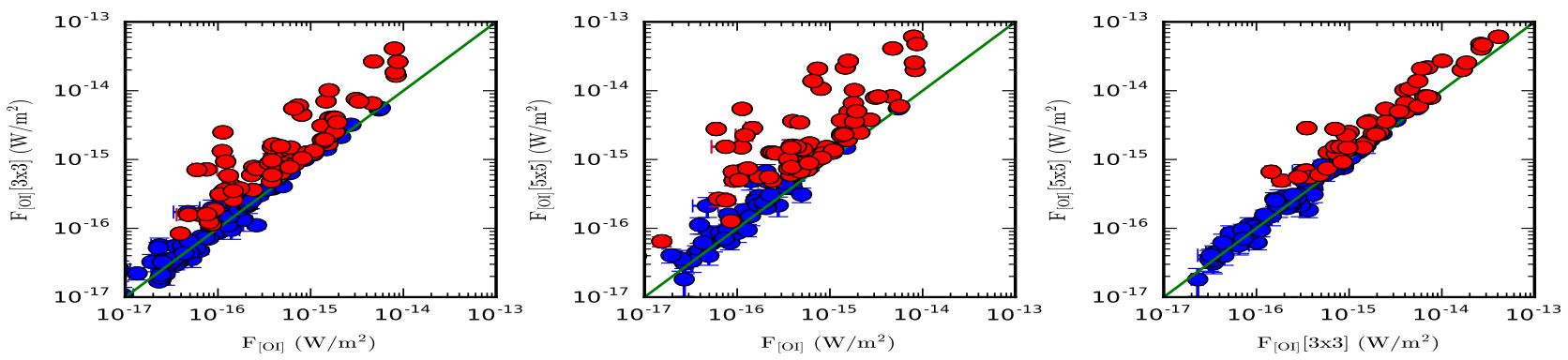

Fig. 4. Extended emission tests for sources in the sample. Red dots identify sources showing extended emission in each of the tests. The solid diagonal line depicts a one-to-one ratio to help identify extended emission.
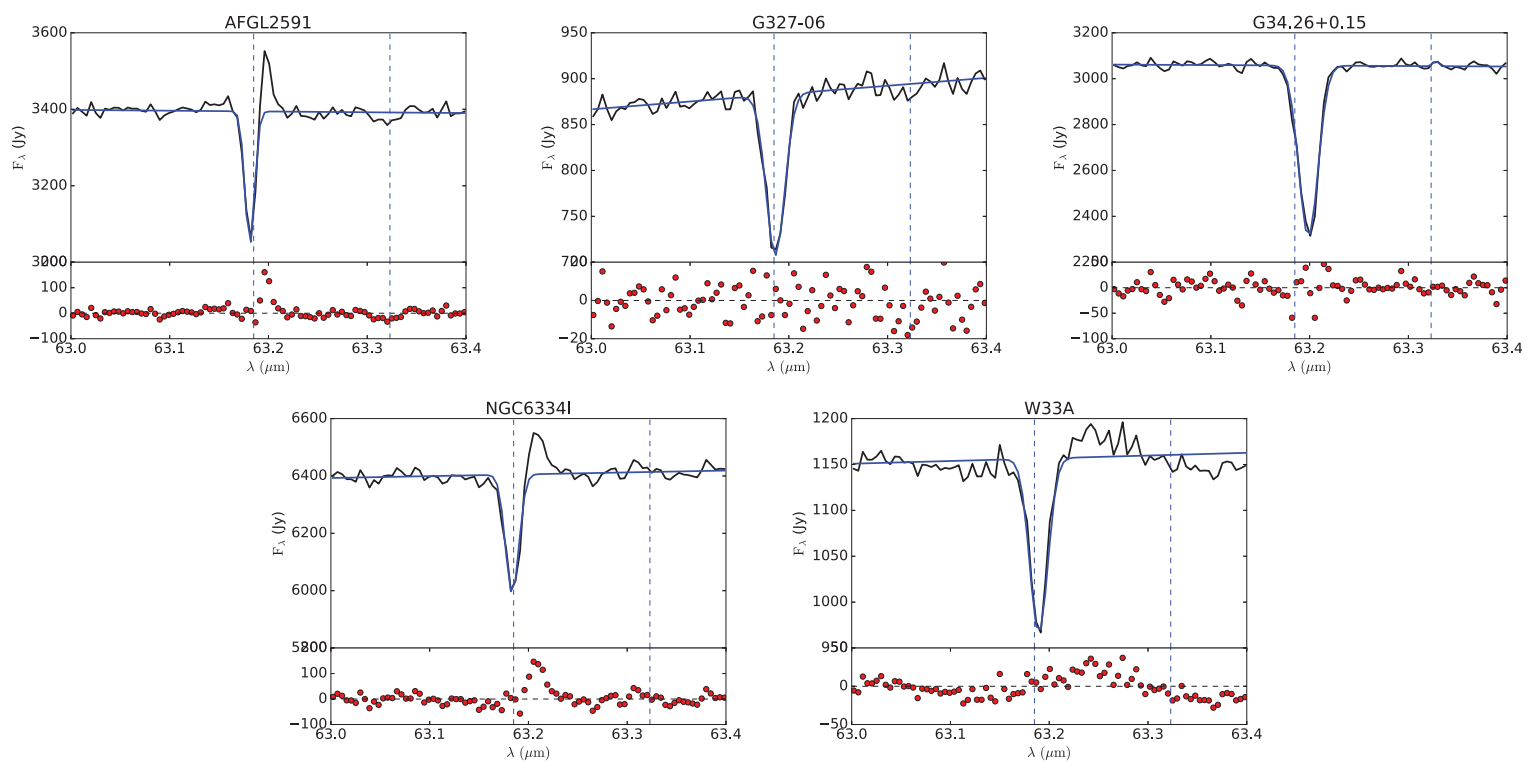

Fig. 5. Spectral profiles for high-mass YSOs showing [OI] line absorption.
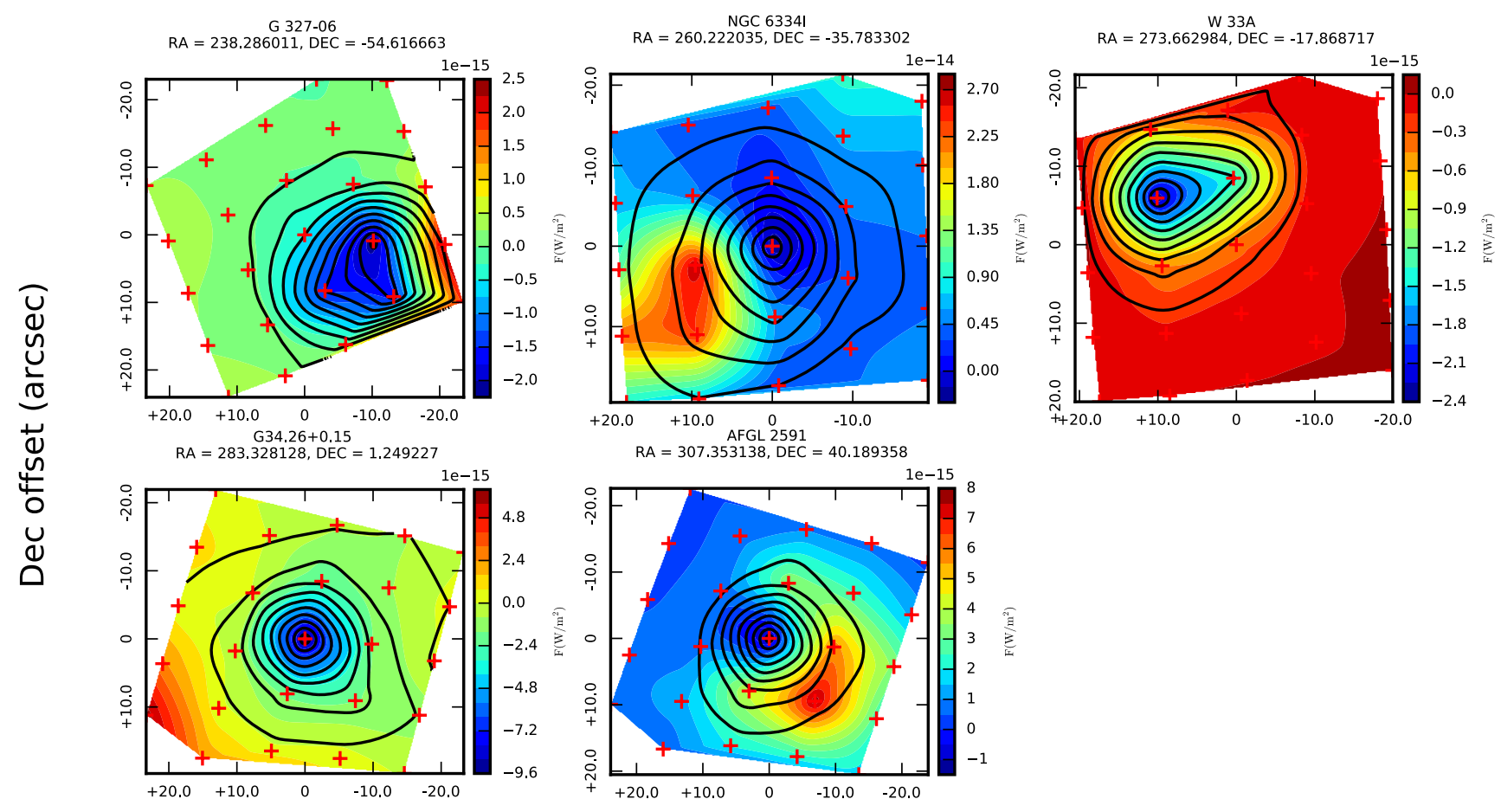

RA offset (arcsec)

Fig. 6. Line emission (coloured contours) and continuum maps at $63 \mu \mathrm{m}$ (solid lines) for sources showing [OI] absorption at $63.185 \mu \mathrm{m}$. The crosses mark the position of individual spaxels. 

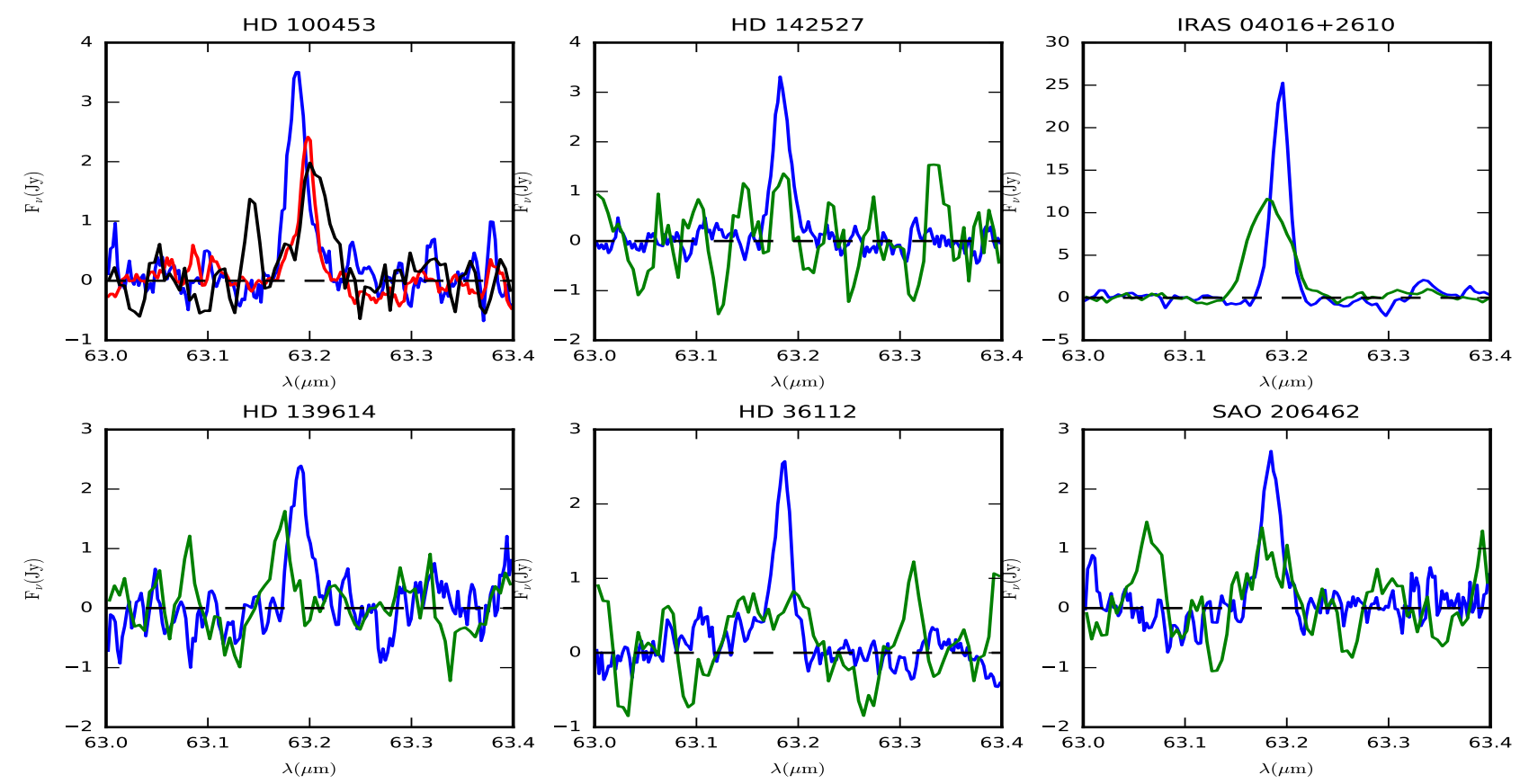

Fig. 7. Spectra obtained at different epochs showing hints of line variability.

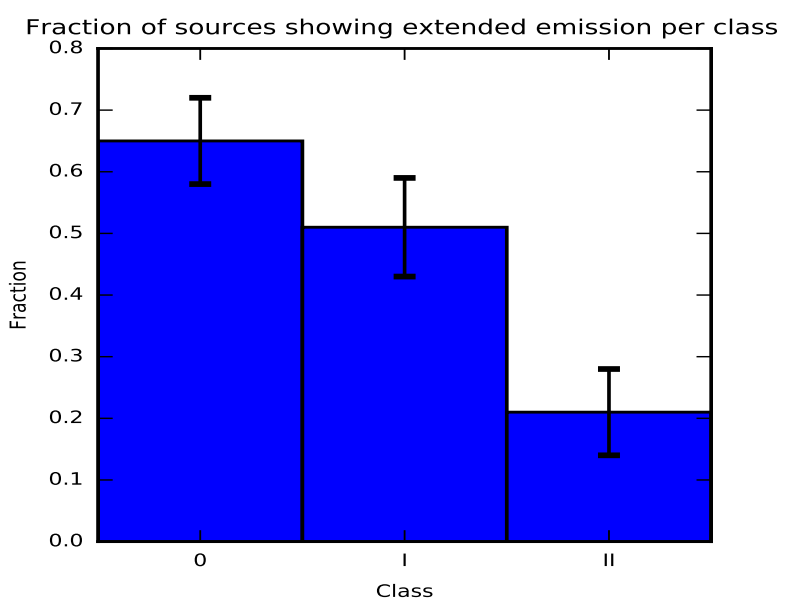

Fig. 8. Bar plot showing the global fraction of sources with extended [OI] emission per class.

protostar (RCrA-IRS7A). Finally, 51 sources showed extended emission when Test 3 was used, 41 of which belonged to Class 0 and I, eight belonged to Class II and transitional, and two are highly embedded protostars (RCrA-IRS7A and AFGL 2591, which shows absorption in the central spaxel, together with extended emission in the surrounding spaxels). The final fraction of extended sources per class is shown in Fig. 8.

For all the sources showing higher fluxes from co-added spaxels, we used the method by Podio et al. (2012) to detect extended emission. This method compares the ratio of line to continuum emission in the different spaxels with that in the central one, aiming to detect residual emission over the expected value. The method implicitly assumes that the continuum is emitted by a point source. While this is true for most Class II sources and transitional discs, it might not be true for Class 0 and I sources in the sample, as shown by Lindberg et al. (2014).

We detected $3 \sigma$ residual emission in the maps of 71 sources (line emission maps for these sources are shown in Fig. A.1 and residual maps are shown in Fig. B.1). FS Tau and FS Tau B are both included in the same OBSID, making the algorithm identify false residual emission. The residual found westward of the source is indeed emitted by FS TauB. The fields for RCrAIRS7B are too crowded, and therefore we decided to exclude them from the analysis. Lindberg et al. (2014) performed a detailed study of this region, and we refer to this paper for more detailed results.

To compare between continuum and line emission, we distinguished three groups of maps. In the first group, we included sources that showed the peaks of line and continuum emission at the same position. In total, 39 sources were included in this first group. Prominent examples of this type are the maps of sources such as IRAS $03235+3004$ or IRAS $04264+2433$. In the second group, we included 18 maps where continuum and line emission peak at different positions. Examples of this sources are NGC 1333 IRAS 4A and VLA 1623-243. Finally, the third group, with 14 observations, included very complex maps, mostly due to the presence of multiple sources. For this third group, the analysis of line emission and continuum maps is precluded, since it is a very complicated task to isolate the contribution of each component. Again, we refer to Lindberg et al. (2014) for insight into the methods that can be used.

Only one source showed extended emission in $\mathrm{o}-\mathrm{H}_{2} \mathrm{O}$ at $63.325 \mu \mathrm{m}$, NGC 2071, a Class 0 source from the WISH program. Its line emission and residual maps are shown in Fig. 9. The source shows both $[\mathrm{OI}]$ and $\mathrm{o}-\mathrm{H}_{2} \mathrm{O}$ residual emission. [OI] residual emission is found south and north-east of the source, while $\mathrm{o}-\mathrm{H}_{2} \mathrm{O}$ residual emission is found southward only. Interestingly, [OI] residual emission is brighter in the northeast position than in the south position, and the maximum is $\sim 10$ times brighter than that of the $\mathrm{o}-\mathrm{H}_{2} \mathrm{O}$ residual. Melnick et al. (2008) detected extended warm $\mathrm{H}_{2} \mathrm{O}$ emission in the region aligned with the direction of the outflow.

\subsection{Multiple components}

van Kempen et al. (2010b) showed that the profile of the [OI] line at $63 \mu \mathrm{m}$ towards HH 46 observed with PACS consisted 


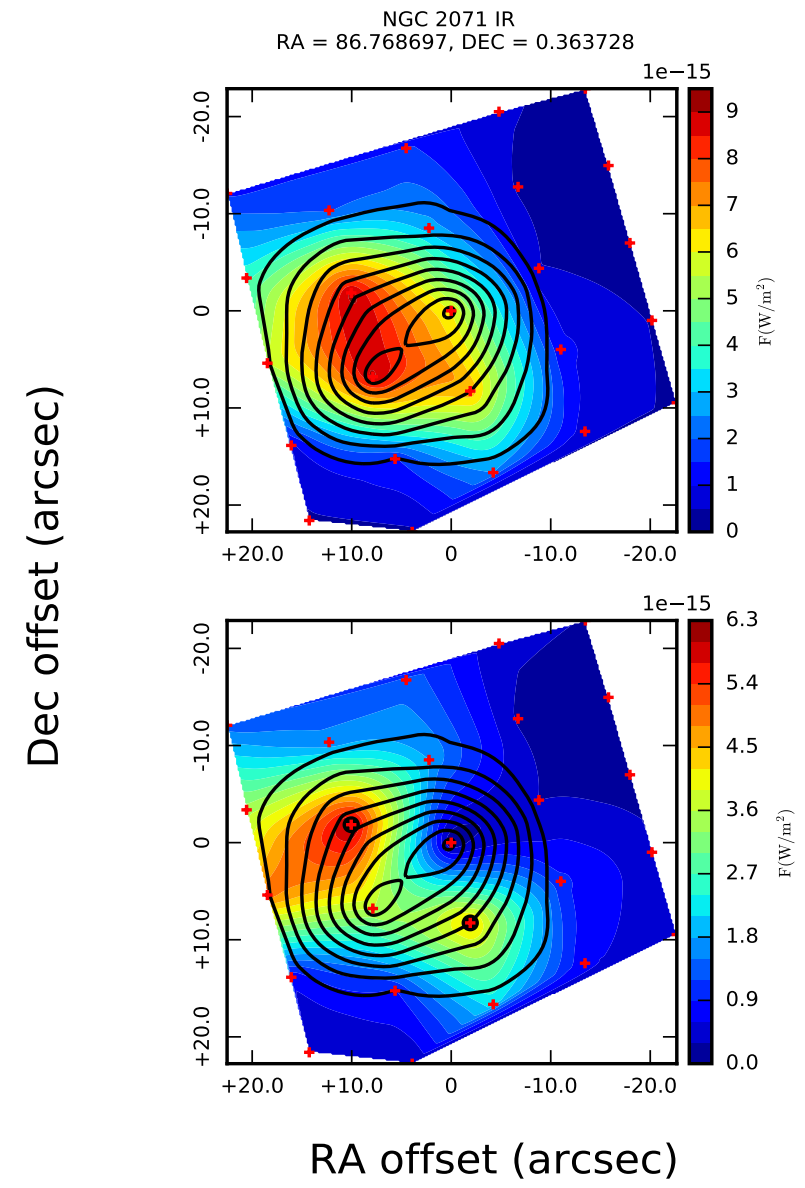

Fig. 9. Top: o- $\mathrm{H}_{2} \mathrm{O}$ emission at $63 \mu \mathrm{m}$ map for NGC 2071 IR. Bottom: residual map for o- $\mathrm{H}_{2} \mathrm{O}$ emission at $63 \mu \mathrm{m}$ for NGC 2071 IR. The solid line contours depict the continuum emission in both panels.

of three components: blue- and red-shifted components and a rest-frame velocity component. The authors also showed that the velocities of the red- and blue-shifted components are consistent with observations of jets in the near-IR and in the optical. In Riviere-Marichalar et al. (2015), the [OI] emission line was fitted by multiple Gaussians in DK Cha, indicating that several dynamical components might be present. The different velocities can be attributed to different components. While the restframe emission seems to be associated with an envelope and/or disc plus wind emission, the most natural explanation for highvelocity components is jet emission.

We performed a multiple Gaussian analysis of the YSOs in the sample. Model fits with one, two, or three Gaussian components were compared using the Bayesian information criterion (BIC, see Feigelson \& Babu 2012) to detect the most representative one. The BIC is described for each model as

$B I C=-2 \times \ln \left(L^{0}(M)\right)+k \times \ln (N)$

where $L^{0}(M)$ is the highest value of the likelihood distribution, $k$ is the number of free parameters, and $N$ the number of spectral points. To detect the best model in each case, we performed a $\chi^{2}$ minimization, and therefore Eq. (1) becomes

$B I C=\chi_{0}^{2}+k \times \ln (N)$

where $\chi_{0}^{2}$ is the lowest value of $\chi^{2}$, corresponding to the highest value of the likelihood distribution. To decide which model better reproduces the observations, the BIC for one-, two-, and three-Gaussians models must be compared. A BIC difference $2<\Delta B I C<6$ shows evidence against the model with the higher BIC, while a BIC difference larger than 10 excludes the model with the higher BIC with high probability.

Thirty sources showed evidence of multiple components. We show in Fig. 10 the resulting fits for these sources, and the Gaussian parameters are given in Table 4. It is a known effect that when a source is not properly centred on a PACS spaxel it can result in a shift in the line centre and in a distortion of the Gaussian shape. However, 24 of the 30 sources are properly centered on their spaxels, and therefore, at least for them, we are sure that the effect is real and not an observational artefact. The number of sources per evolutionary stage is as follows: one is a highly embedded source with unknown evolutionary stage, four are Class 0 sources, one is an intermediate Class 0/I sources, 12 are Class I, one is an intermediate Class I/II, 10 are Class II (six are T Tauri and four are HAeBe stars), and one is a transitional disc.

Fourteen of them have a smaller separation between the different components than the spectral resolution at $63 \mu \mathrm{m}$, $\sim 88 \mathrm{~km} \mathrm{~s}^{-1}$. Twenty sources are better reproduced by a model with two Gaussians, while twelve sources are better reproduced by a model with three components. The most prominent case is that of DK Cha (see Riviere-Marichalar et al. 2015), where the separation between the different components in the central spaxel is evident. Of the 30 sources that need multiple Gaussians to be fitted, 21 show evidence of extended emission according to their $3 \times 3$ fluxes and $5 \sigma$ residual emission in their IFUs.

To test wether detecting multiple components was linked to high $\mathrm{S} / \mathrm{N}$ observations, we compared the distributions of continuum and line $\mathrm{S} / \mathrm{Ns}$ of the whole sample with that of sources that are better reproduced by multiple Gaussians. Histograms comparing the distributions are shown in Fig. 11, demonstrating that multiple-Gaussian detections are linked to high $\mathrm{S} / \mathrm{N}$ sources. A Kolmogorov-Smirnov test confirmed this trend: the probability that both distributions are drawn from the same sample are $\sim 10^{-3}$ for the continuum $\mathrm{S} / \mathrm{N}$ and $\sim 10^{-7}$ for the line $\mathrm{S} / \mathrm{N}$. Therefore, we cannot rule out that some, if not all, of the low$\mathrm{S} / \mathrm{N}$ sources also have multiple components.

\section{Discussion}

As shown by Podio et al. (2012), [OI] emission can be extended along the jet direction, indicating that the jet contributes to, or even dominates, the line emission. We computed in Sect. 4.4 residual emission maps for sources in the sample, and showed that $[\mathrm{OI}]$ is extended for a large number of sources: 83 sources show extended emission when the flux from the central spaxel is compared to that of co-added ones, and 71 of them showed residual emission. We also showed that $\mathrm{H}_{2} \mathrm{O}$ emission is extended in only one case, compared to [OI], an indication that the o$\mathrm{H}_{2} \mathrm{O}$ line most likely has its origin in a compact and high-density region at the base of the jet (see Podio et al. 2012), like the disc or shocks along the cavity walls in the envelope. Aiming to better understand the physics behind the line emission at $63 \mu \mathrm{m}$ and its origin, we compared line fluxes and different observables. To minimize the scatter associated with the different distances, all the fluxes where scaled to the distance to Taurus (140 pc, Kenyon et al. 2008), so that only sources belonging to known associations or with known distances were used. The distances for the different associations are given in Table 2. 
P. Riviere-Marichalar et al.: Herschel-PACS observations of far-IR lines in young stellar objects. I.
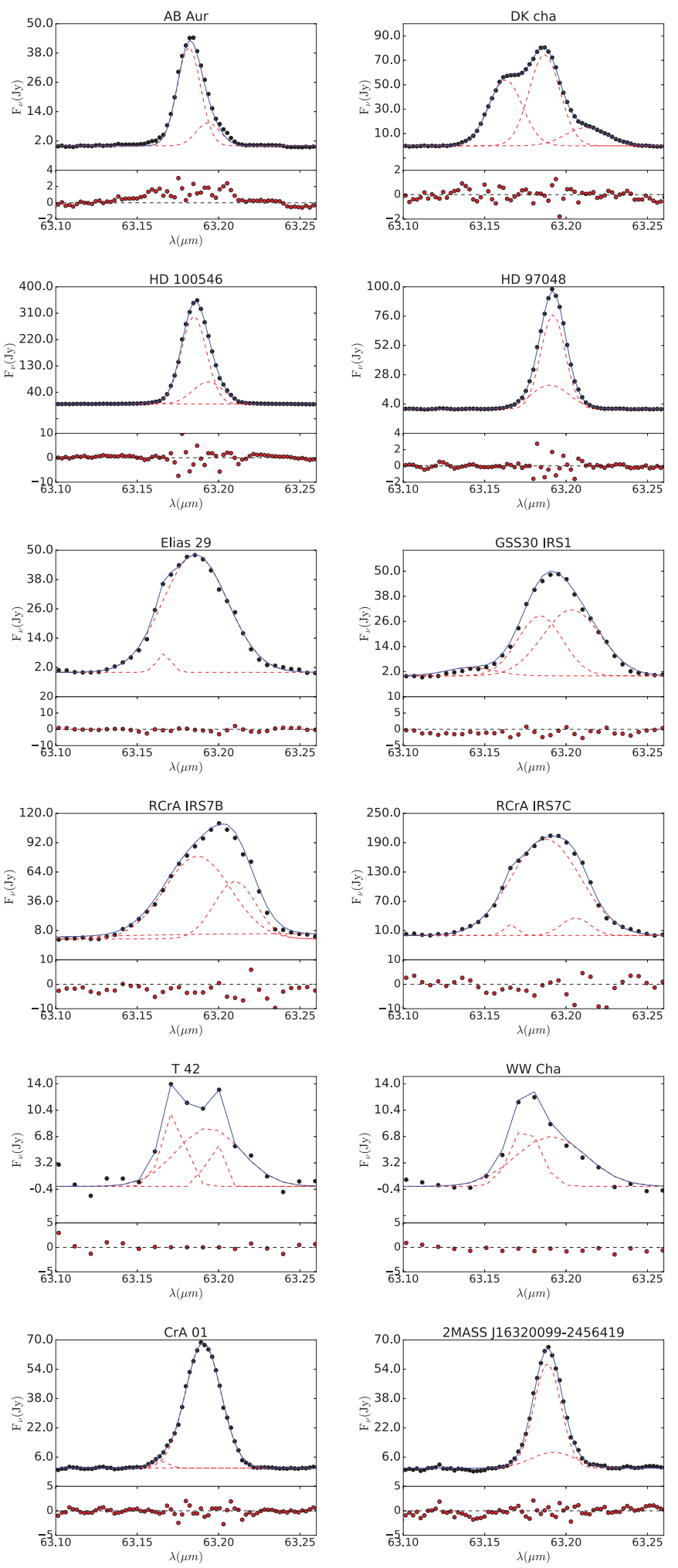
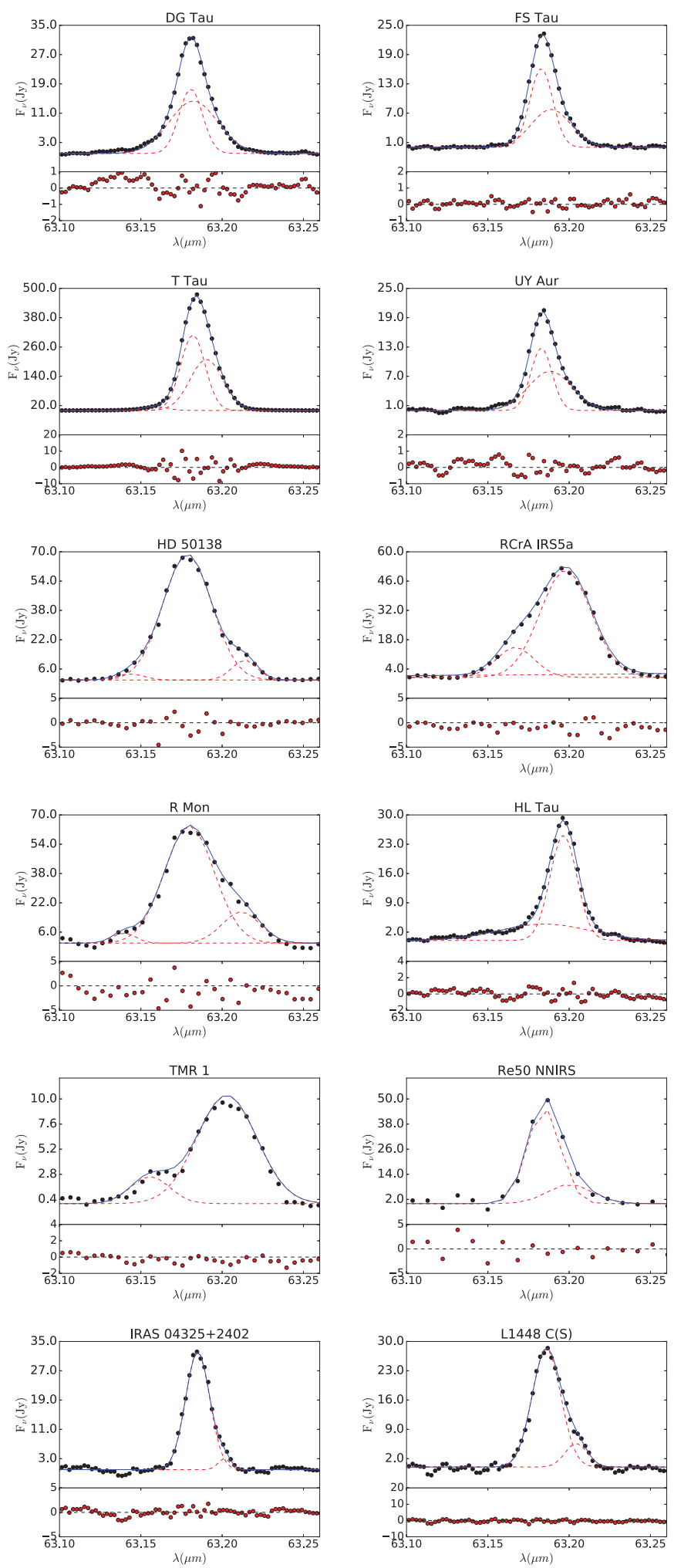

Fig. 10. Multiple-component fits for sources that are better fitted by a combination of two to three Gaussians. The red dashed lines show the individual Gaussian components, while the blue solid lines depict the combined model. Black dots represent the observed spectra.

\subsection{Correlation between far-IR line emission fluxes}

In Fig. 13, we show the relation between o- $\mathrm{H}_{2} \mathrm{O}$ line flux and [OI] line flux at $63 \mu \mathrm{m}$. This correlation was previously found by Riviere-Marichalar et al. (2012b). With better source statistics, we now tentatively see a change in slope from Class 0 and
I to Class II sources. However, the small number of $\mathrm{o}-\mathrm{H}_{2} \mathrm{O}$ detections precludes any firm conclusion. The fact that $[\mathrm{OI}]$ and o- $\mathrm{H}_{2} \mathrm{O}$ fluxes at $63 \mu \mathrm{m}$ are correlated might suggest a common origin for the two lines lines. However, while [OI] is sometimes extended, the o- $\mathrm{H}_{2} \mathrm{O}$ line is extended in only one source. Furthermore, while we sometimes need multiple components to 

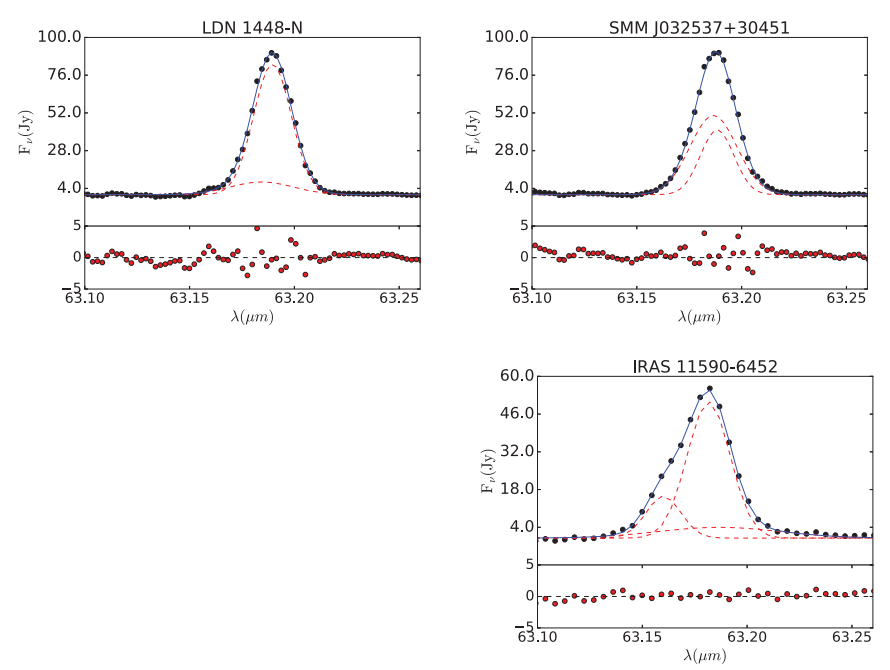

Fig. 10. continued.

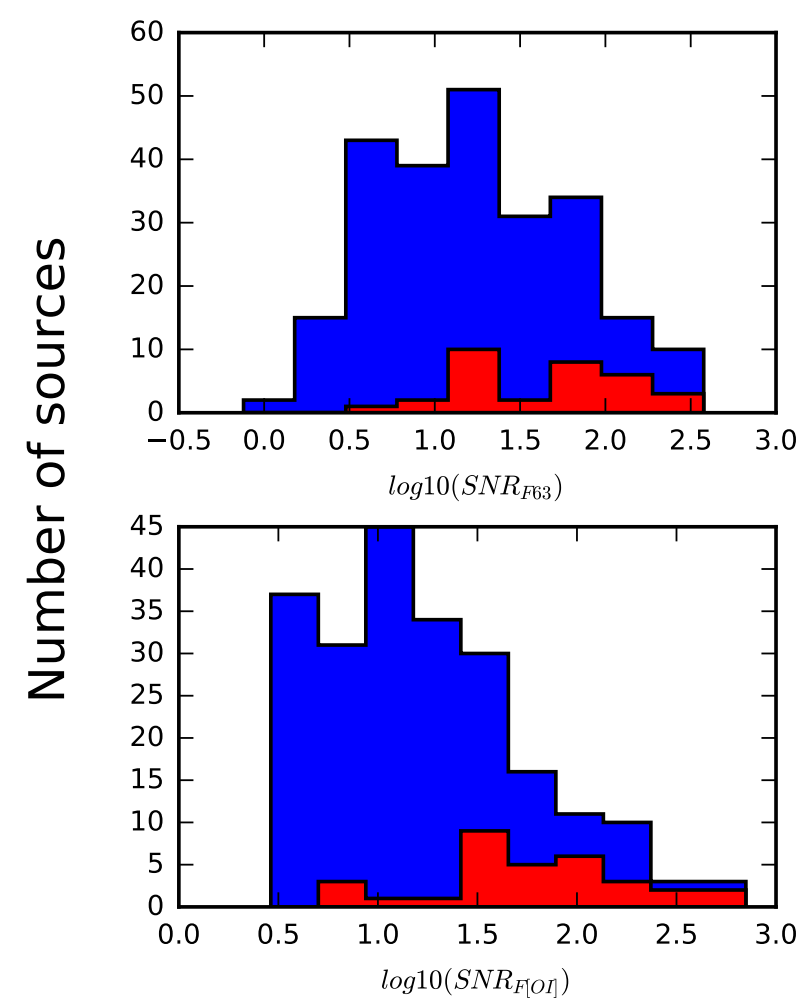

Fig. 11. Top: distribution of continuum $\mathrm{S} / \mathrm{N}$ for the whole sample (blue) and for sources that are better reproduced by multiple Gaussians (red). Bottom: distribution of line $\mathrm{S} / \mathrm{N}$ for the whole sample (blue) and for sources that are better reproduced by multiple Gaussians (red).

fit the [OI] line, a simple Gaussian fit is enough to fit the o$\mathrm{H}_{2} \mathrm{O}$ line in all detections. However, the lack of multiple components can be linked to the low-S/N nature of the detections. Adding more evidence against a co-spatial origin, the critical density for the $\mathrm{o}-\mathrm{H}_{2} \mathrm{O}$ line is orders of magnitude higher than the one for $[\mathrm{OI}]$.

The lack of multiple-components in the profile of $\mathrm{o}-\mathrm{H}_{2} \mathrm{O}$ might be due to low $\mathrm{S} / \mathrm{N}$. o- $\mathrm{H}_{2} \mathrm{O}$ detections with a line flux $\mathrm{S} / \mathrm{N}$ similar to that of $[\mathrm{OI}]$ lines that require multiple Gaussians (such as L1448-C(S), $S / N_{\mathrm{fH}_{2} \mathrm{O}} \sim 24$ ), are well reproduced by a single Gaussian. We show in Fig. 12 a comparison of the [OI]
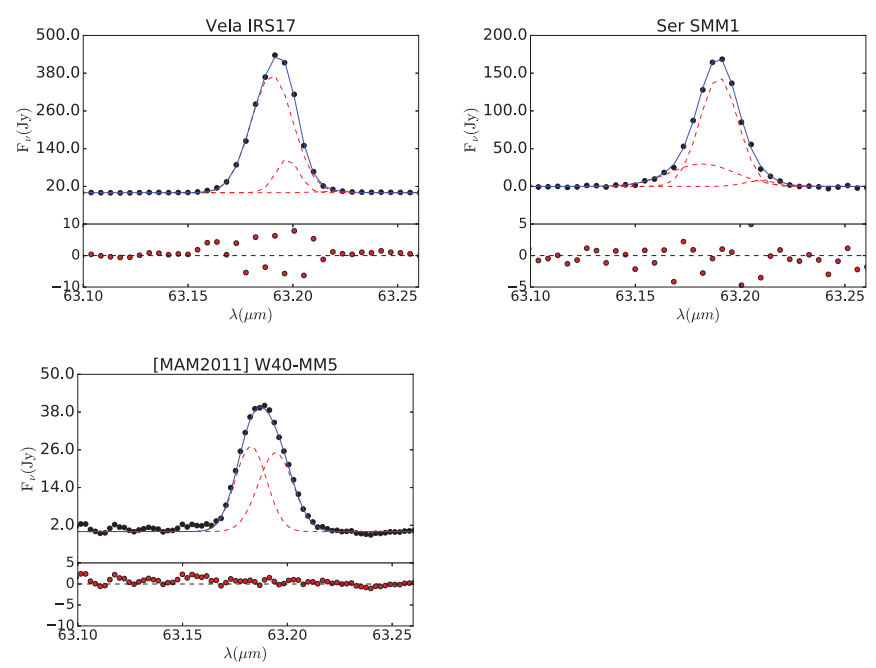

Table 2. Distances to associations in the sample.

\begin{tabular}{lcc}
\hline \hline $\begin{array}{l}\text { Association } \\
-\end{array}$ & $\begin{array}{c}\text { Distance } \\
(\mathrm{pc})\end{array}$ & $\begin{array}{c}\text { Reference } \\
-\end{array}$ \\
\hline BPMG & 33 & Zuckerman \& Song (2004b)* \\
Cha & 165 & Luhman (2008) \\
Cha II & 178 & Luhman (2008) \\
CrA & 130 & Neuhäuser \& Forbrich (2008) \\
$\eta$ Cha & 97 & Mamajek et al. (1999) \\
Lupus & 150 & Comerón (2008) \\
Lupus III & 200 & Comerón (2008) \\
Oph & 130 & Wilking et al. (2008) \\
Per & 235 & Hirota et al. (2008) \\
Serpens & 415 & Dzib et al. (2010) \\
Taurus & 140 & Kenyon et al. (2008) \\
TWA & 50 & Webb et al. (1999) \\
Tuc Hor & 46 & Zuckerman \& Song (2004b) \\
Up Sco & 145 & de Zeeuw et al. (1999) \\
\hline
\end{tabular}

Notes. ${ }^{(*)}$ The distance provided is the average of the distances to individual sources shown in the referenced papers.

and $\mathrm{H}_{2} \mathrm{O}$ line profiles for sources whose [OI] profiles are better reproduced by a combination of Gaussians. L1448-C(S) and 2MASS J16320099-2456419 show similar [OI] and $\mathrm{H}_{2} \mathrm{O}$ profiles, with bumps at similar velocities $\left(\sim 50-100 \mathrm{~km} \mathrm{~s}^{-1}\right)$, but narrower $\mathrm{H}_{2} \mathrm{O}$ lines. By contrast, L 1448N, FS Tau and UY Aur show very different profiles. $\mathrm{T}$ Tau show similar shapes for both lines, but the $\mathrm{H}_{2} \mathrm{O}$ shows a narrower profile and no bumps are seen. Observations with high spectral resolution are needed to explain multiple components.

The most likely explanation for the spatial extension of the emission, and for the presence of multiple components in the [OI] line is a contribution from jet emission associated with the source (Podio et al. 2012; Howard et al. 2013). The high-velocity components $\left(v_{\mathrm{HVC}} \sim 100 \mathrm{~km} \mathrm{~s}^{-1}\right)$ must be associated with jets, while the low-velocity ones (which might also be rest-frame velocity, due to the limited spectral resolution) can be associated with envelope and wind emission, as well as disc emission.

$\mathrm{o}-\mathrm{H}_{2} \mathrm{O}$ seems to be dominated by disc or envelope emission or compact jet emission, since extended emission is observed in only one Class I source, and the line profiles are consistent 
P. Riviere-Marichalar et al.: Herschel-PACS observations of far-IR lines in young stellar objects. I.
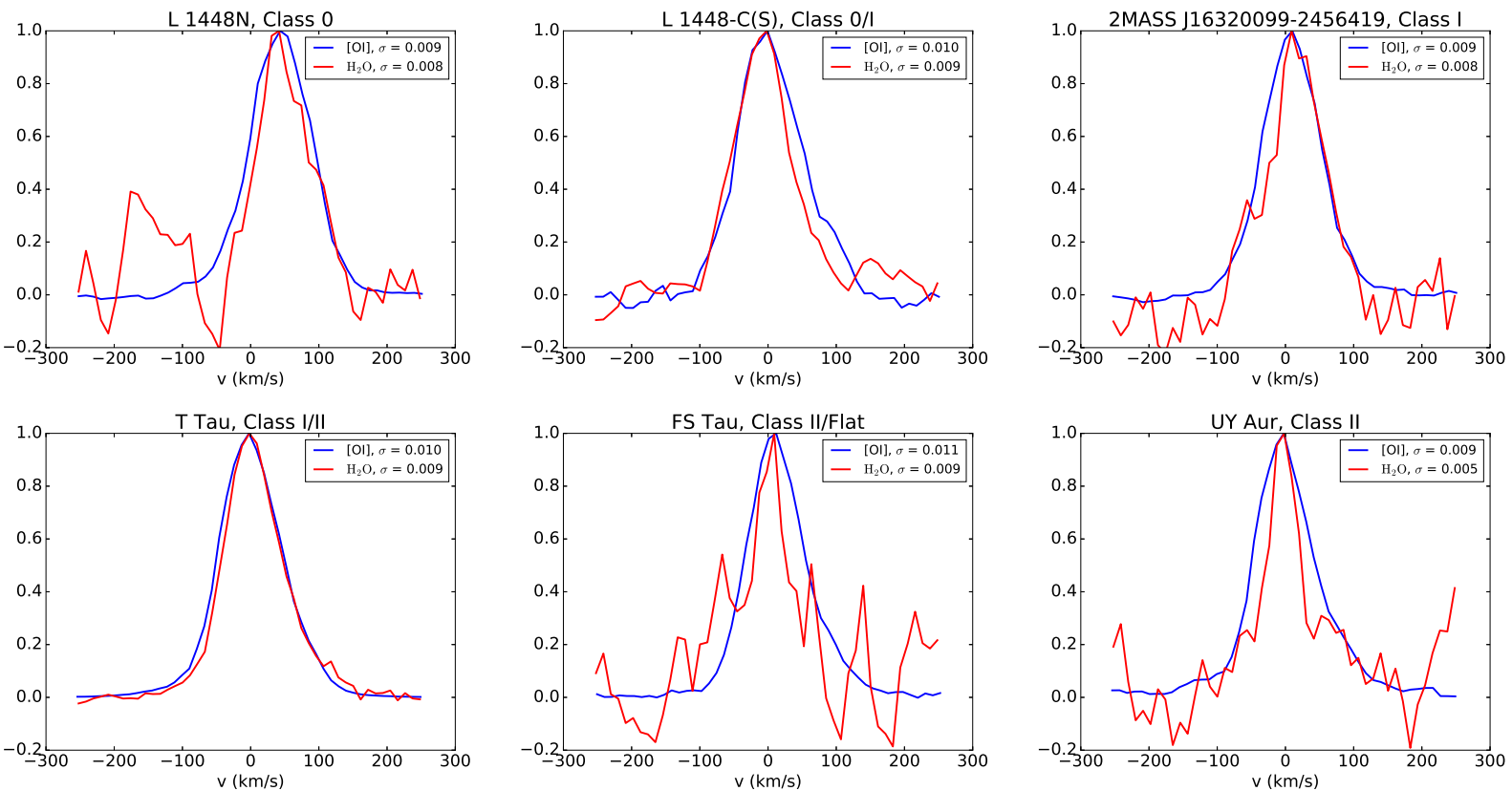

Fig. 12. Comparison of the $[\mathrm{OI}]$ and $\mathrm{o}-\mathrm{H}_{2} \mathrm{O}$ line profiles for sources whose $[\mathrm{OI}]$ profiles are better reproduced by multiple Gaussians. In the legends we show the measured $\sigma$ of the Gaussian fits.

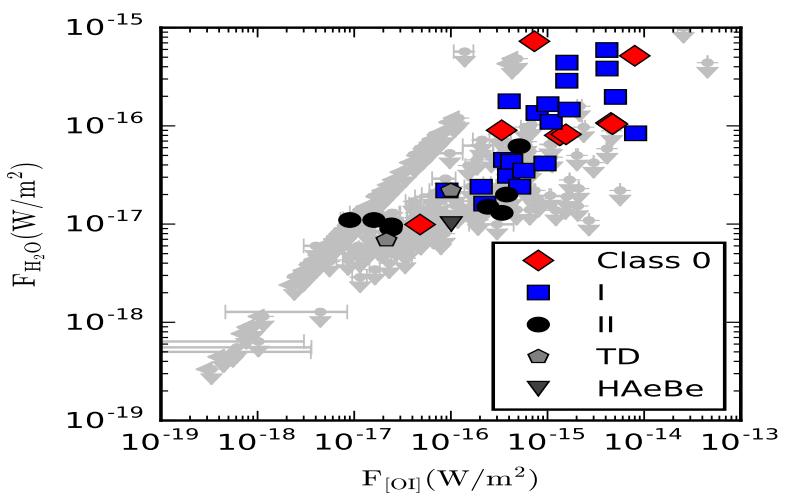

Fig. 13. o- $\mathrm{H}_{2} \mathrm{O}$ line flux versus [OI] line flux at $63 \mu \mathrm{m}$. Arrows show the positions of upper limits for non-detected sources. All fluxes have been scaled to the distance of Taurus.

single Gaussians. However, we mostly detected water emission in jet sources, and therefore we cannot rule out that the emission originates at the base of the jet, and that the lack of multiple components is due to a low $\mathrm{S} / \mathrm{N}$ in the observations presented here.

\subsection{Correlations with continuum emission}

Howard et al. (2013) studied [OI] emission in Taurus and found a correlation between [OI] line emission at $63 \mu \mathrm{m}$ and the continuum at the same wavelength. This correlation was later confirmed for other associations (Mathews et al. 2013; Riviere-Marichalar et al. 2015). We have extended the study to the entire sample of YSOs observed with PACS in spectroscopic mode. The resulting plot is shown in Fig. 14. The different sources are placed at different loci in the diagram. Class 0 and I sources show on average higher line fluxes for the same continuum level than Class II and transitional discs. Furthermore, transitional discs show also lower [OI] fluxes for the same level of continuum emission than Class II sources. Transitional discs
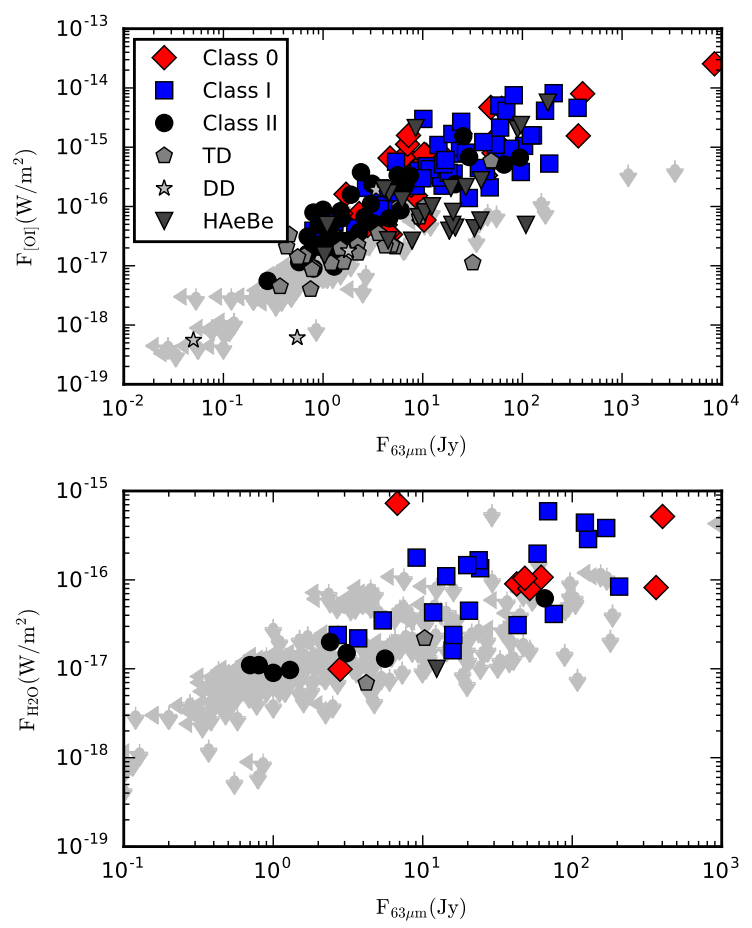

Fig. 14. [OI] (top) and o- $\mathrm{H}_{2} \mathrm{O}$ (bottom) line fluxes versus continuum flux at $63 \mu \mathrm{m}$ for sources in the sample. Sources are labelled according to evolutionary stage. All the fluxes have been scaled to the distance to Taurus $(140 \mathrm{pc})$. Arrows show the position of upper limits for nondetected sources.

are located in the lowest part of the diagram because theirs is the lowest [OI] emission for the same continuum level. Class II sources show intermediate [OI] fluxes, while the Class 0 and I sources show the highest flux levels for the same continuum.

The correlation between [OI] flux and the continuum flux at $63 \mu \mathrm{m}$ is different for jet and non-jet sources (Howard et al. 2013). Given the correlation between line emission and 

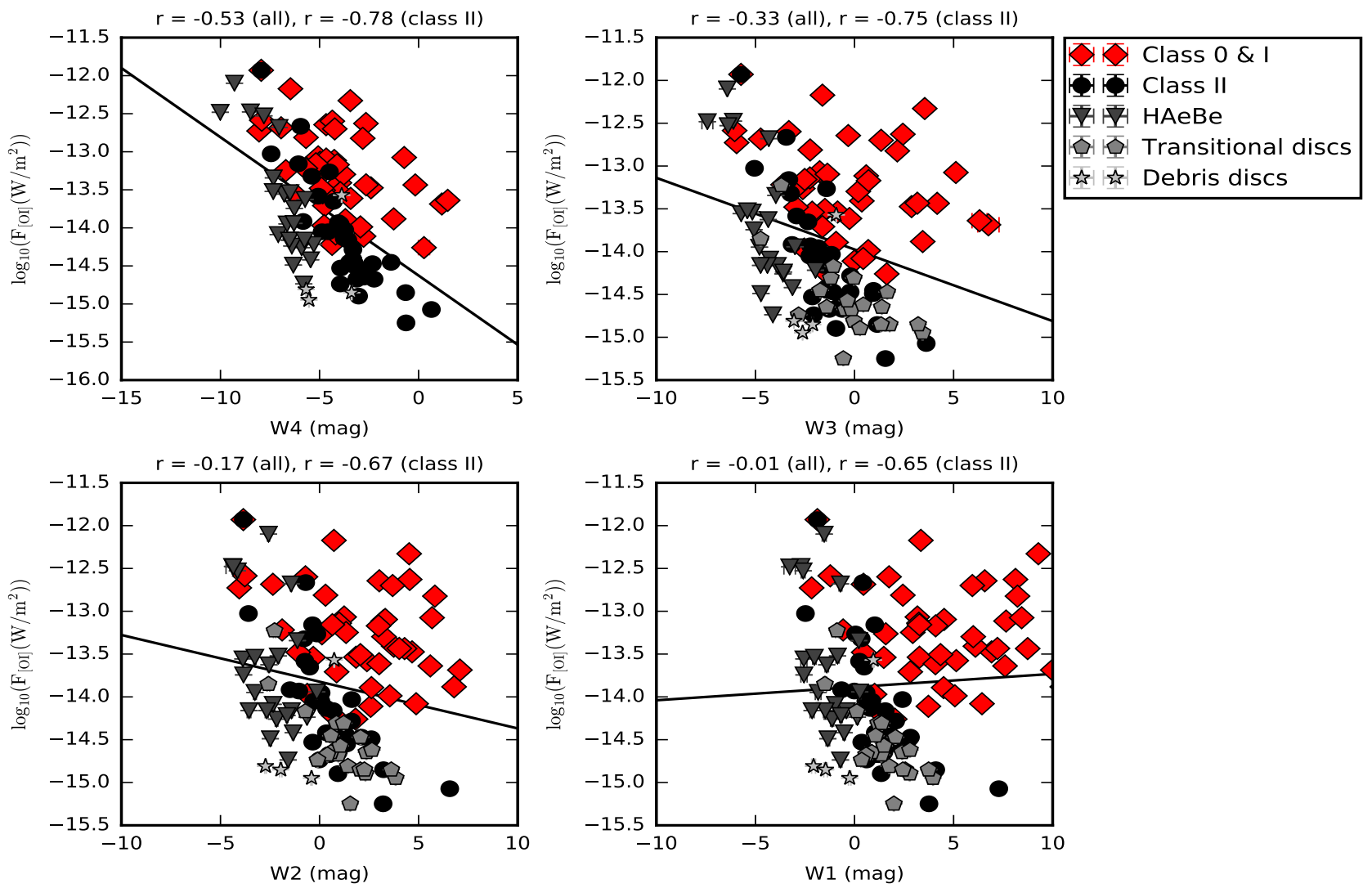

Fig. 15. [OI] fluxes at $63 \mu \mathrm{m}$ versus WISE magnitudes. All the fluxes have been scaled to the distance to Taurus. Magnitudes are corrected for distance. Spearman correlation coefficients are shown at the top of each plot.

continuum emission at $63 \mu \mathrm{m}$, we tested correlations against continuum photometry at different wavelengths. We first started by retrieving WISE (Wright et al. 2010) magnitudes for sources in the sample, within a search radius of $2.6^{\prime \prime}$. When testing the correlations, we only included sources belonging to associations with known distances, or belonging to known associations, to correct the fluxes and magnitudes for distance. All the fluxes were scaled to the distance to Taurus $(140 \mathrm{pc})$, and the magnitudes were converted into absolute magnitudes. The resulting comparison is shown in Fig. 15. We observed clear correlations between the [OI] flux and WISE band 4 flux, but the correlation seems to vanish for WISE1, with a Spearman probability for the null hypothesis (i.e. that there is no correlation) that decreases from $3.4 \mu \mathrm{m}$ (WISE 1) to $22 \mu \mathrm{m}$ (WISE 4). However, the results change when only Class II sources are considered, and the correlation is present for all the WISE bands, with strong to very strong correlation coefficients in the range 0.7 to 0.8 .

The scatter in the correlations has many contributions, such as instrumental uncertainties, scatter in distance within the same association, and different disc mass and geometry. Furthermore, jets and winds also contribute to the [OI] line flux, but not to the continuum, which increases the scatter. We observed clear correlations from 22 to $63 \mu \mathrm{m}$. The fact that [OI] correlates with magnitudes at different IR wavelengths, and that the strength of the correlation increases with wavelength, and is more pronounced for Class II sources very likely indicates that dust at different temperatures and gas emission are related, which most likely points to a contribution from discs and envelopes. However, this is not the only interpretation. Sources accreting at higher rates will show brighter continuum emission at $63 \mu \mathrm{m}$. If accretion is driving [OI] emission at $63 \mu \mathrm{m}$, then sources with higher accretion rates will also show brighter [OI] emission at $63 \mu \mathrm{m}$, explaining the correlation.

\subsection{Comparison with DENT models}

To better understand the correlations, we compared our results with predictions from the DENT grid (Woitke et al. 2010; Kamp et al. 2011). The DENT grid consists of more than $3 \times 10^{5}$ models of protoplanetary discs that were developed to help in the interpretation of photometric and spectroscopic observations of protoplanetary discs for the GASPS program (Dent et al. 2013). The grid contains models representing different evolutionary stages. To restrict the number of models and interpret the results, we fixed $R_{\text {in }}=R_{\text {sub }}$ (where $R_{\text {sub }}$ is the dust sublimation radius), $R_{\text {out }}=300 \mathrm{au}$, a surface mass density distribution power-law with index $\epsilon_{0}=1.0$, the gas-to-dust ratio to 100 and the minimum grain size to $0.05 \mu \mathrm{m}$. We also excluded edge-on models $\left(i=90^{\circ}\right.$ ) and models with $\beta=0.8$ (where $\beta$ is the exponent of the scale height relation $H=H_{0}\left(r / r_{0}\right)^{\beta}$, leaving us with $\beta=1.0$ and $\beta=1.2$ ).

The DENT grid does not include continuum fluxes at $22 \mu \mathrm{m}$, but at $24 \mu \mathrm{m}$, so that this is what we show in Fig. 16. The difference in wavelength is so short that any effect on the shape of the correlation must be small. Owing to the limited parameter space covered by the DENT grid, the brightest sources are not covered by the models. The spearman probability $(p)$ for the null hypothesis (i.e., that there is no correlation) is $p \ll 1.0^{-3}$ for the observations and for the models. We then fitted a straight line in the log-log space to the distributions of models and observations. The resulting fit has slope $m=0.72$ for the observational distribution, and $m=0.61$ for the distribution of models. 
P. Riviere-Marichalar et al.: Herschel-PACS observations of far-IR lines in young stellar objects. I.

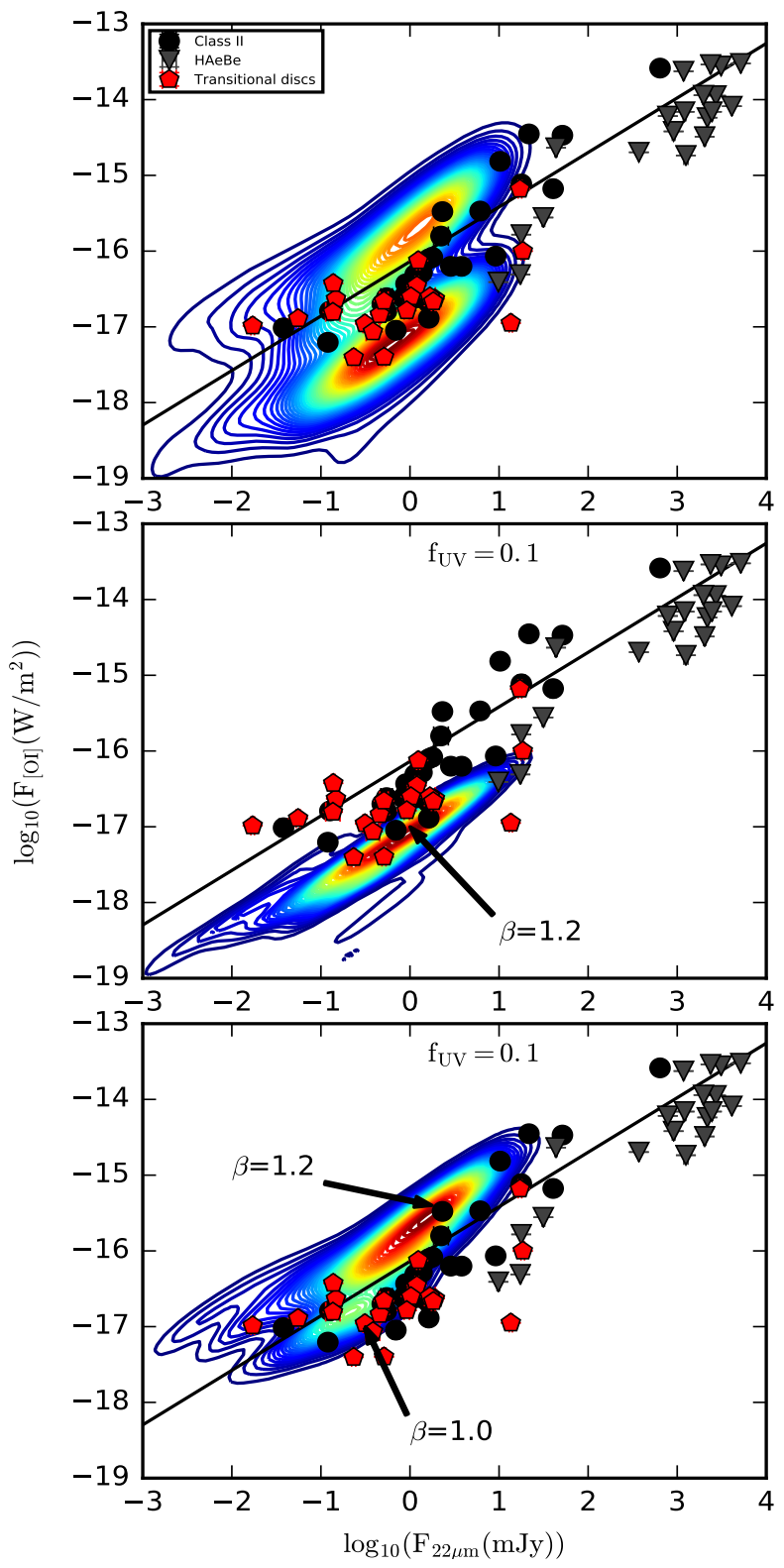

Fig. 16. [OI] fluxes at $63 \mu \mathrm{m}$ versus WISE flux at $22 \mu \mathrm{m}$ : observations compared to models. The contours show the density of points for models from the DENT grid. Black dots show the position of observed Class II sources, red pentagons are transitional discs, and grey triangles are HAeBe stars. The top panel shows the whole distribution of selected models. The middle panel shows the distribution of models with $f_{\mathrm{UV}}=0.1$, while the bottom panel shows models with $f_{\mathrm{UV}}=0.001$. The arrows point to the location of sources with different values of $\beta$. The solid line is a linear fit to the observed data.

According to Woitke et al. (2010) the UV radiation field is one of the main drivers of [OI] emission, together with the flaring geometry and the total gas mass. To better understand the influence of these parameters on the [OI] flux distribution, we performed a more detailed analysis by fixing one of them at a time, and letting the other parameter free. The resulting comparison is shown in Fig. 16. For $f_{\mathrm{UV}}=0.001$, only models with $\beta=1.2$ can reproduce the observations. When $f_{\mathrm{UV}}=0.1$, models with $\beta=1.2$ overestimate the flux for most of the observations. It is clear from the plots that we need intermediate values of both $f_{\mathrm{UV}}$ and $\beta$. However, extremely flared discs with low $f_{\mathrm{UV}}$ or flat discs with very high $f_{\mathrm{UV}}$ overlap with the observations.
Overall, DENT models provide a good description of [OI] emission at $63 \mu \mathrm{m}$ for Class II sources. This shows that observations of Class II sources are compatible with pure protoplanetary disc models. Furthermore, the DENT models do not include jets or outflows, but they describe the emission well. The theoretical prediction by Woitke et al. (2010) that in a disc $f_{\mathrm{UV}}$ and $\beta$ control $[\mathrm{OI}]$ emission is therefore compatible with our observations. We conclude that disc models provide an explanation for the correlations, but other solutions cannot be excluded.

\subsection{Origin of line emission}

In a study of molecular and atomic emission towards $\mathrm{HH} 46$, van Kempen et al. (2010b) showed that the bulk of [OI] emission comes from low-velocity gas, after the impact of highvelocity jets in the cavity walls. The authors also concluded that the high-velocity component observed in the central and outer spaxels originates in fast dissociative shocks in the lower density jet. Podio et al. (2012) demonstrated that atomic [OI] and [CII] line emission were extended and correlated with the direction of optical jets, and proposed that the extended atomic emission could be produced by J-shocks. Karska et al. (2013) studied a sample of low-mass Class 0 and I YSO and concluded that [OI] emission at $63 \mu \mathrm{m}$ originates in dissociative shocks. The authors also distinguished two groups of sources with extended emission, based on morphological differences: a compact group, where $[\mathrm{OI}]$ and $\mathrm{OH}$ emission dominates the central spaxel, while $\mathrm{CO}$ and $\mathrm{H}_{2} \mathrm{O}$ can follow the same trend or are dominated by offsource emission, and an extended group, where $\mathrm{OH}$ off-source emission is strong. Class 0 sources dominate the extended group while Class 0 and I are equally represented in the compact group. The ratios of [OI] line emission at 63 and $145 \mu \mathrm{m}$ computed by Lee et al. (2014) for six low-mass embedded sources in Taurus were consistent with an origin in C-shocks, and again the authors highlighted that atomic emission is commonly extended along the jet direction. Additional support for a jet origin comes from Nisini et al. (2015), where the very similar profiles shown by $[\mathrm{OI}]$ and $[\mathrm{FeII}]$ towards LDN $1448 \mathrm{~N}$ were highlighted. The authors were able to separate the contributions from the different dynamical components in a few favourable cases, and observed an increment in [OI] velocity with distance from the central source, in agreement with observations of $\mathrm{SiO}$.

In the present paper we have studied the spatial distribution of [OI] line emission towards 110 Class 0 and I sources, and detected hints of extended emission in at least 60 of them $(55 \% \pm 5)$. When separated by groups (see Fig. 8$)$, we found a slightly larger extended emission fraction for Class $0\left(0.63_{-0.08}^{+0.07}\right)$ than for Class I $(0.51 \pm 0.07)$, although they are compatible within the errors. The fraction then dramatically decreases for Class II sources $(0.17 \pm 0.08$, where we included Class II, both HAeBe and $\mathrm{T}$ Tauri, and transitional discs). We interpret this as a clear evidence that extended emission in Class 0 , I, and II sources is due to shocks along the jet direction, in agreement with the results discussed in the previous paragraph, and that outflow activity drops with age.

Additional evidence for a jet contribution comes from the fact that 30 sources needed multiple Gaussians to fit the line profile. The reason for the low number is that only the most favorable cases will result in broad complex profiles because of the limited spectral resolution of PACS. Therefore, it is clearly established that extended emission is due to a jet contribution, and that at least part of the compact emission is also due to shocks at the base of the jet, near the compact source. 
However, various authors explained [OI] emission in Class II sources solely by means of disc emission (see, e.g. Thi et al. 2010; Woitke et al. 2011; Meeus et al. 2010; Thi et al. 2013, 2014; Tilling et al. 2012, among others). Early results by Mathews et al. (2010) ruled out an outflow shock origin for the [OI] line emission observed towards HD 169142 and showed that TWA 01 and RECX 15 observations required extreme outflowing fractions to explain [OI] emission through outflow activity. Furthermore, the authors highlight edthat there is no evidence of outflows in these sources, and the spectral profiles for RECX 15 and TWA 01 are not resolved, precluding the presence of jets with line-of-sight velocities higher than $45 \mathrm{~km} \mathrm{~s}^{-1}$. Gorti et al. (2011) modelled in detail a large set of emission lines towards TWA 01, from UV to radio emission, and concluded that [OI] line emission comes from a region that covers almost the whole disc (30-120 au). The comparison of [OI] line emission versus continuum emission at $63 \mu$ m performed by Howard et al. (2013) for Taurus sources leads to different loci for jet sources and sources without known jets. Jet sources show fluxes up to 20 times larger than than the non-jet sources. Furthermore, the authors discussed that transitional discs show even fainter fluxes, a trend that was confirmed by Keane et al. (2014), who studied [OI] emission in 17 transitional discs and demonstrated that they show weaker emission than full Class II discs, tentatively attributing the difference to flatter and/or less massive transitional discs compared to full ones.

In Sect. 5.3 we have shown that radiative transfer models of protoplanetary discs can explain [OI] emission in Class II sources, adding evidence for a disc contribution. Gorti \& Hollenbach (2008) showed that [OI] emission can be emitted by the surface of protoplanetary discs at all radii. Protoplanetary disc modelling of HAeBe stars by Kamp et al. (2010) predicted [OI] emitting regions extending from 30 to $100 \mathrm{au}$. Correlations between line emission and the continuum emission from 4.6 to $63 \mu \mathrm{m}$ shown in Sect. 5.2 argue in favour of an extended emitting region, and for similar spatial origins for the line and continuum emission, additionally supporting the likeliness of a disc contribution. Transitional discs in Fig. 14 lie in the lower envelope of the cloud of points. We consider that they probably represent the real disc contribution, since no strong jet activity is expected in these sources.

Overall, there is strong observational evidence supporting contributions from both the jet (in Class 0, I and II sources) and the disc (Class II sources), while van Kempen et al. (2010b) ruled out a contribution from the passively heated envelope present in Class 0 and I sources.

\section{Summary and conclusions}

We have compiled Herschel-PACS observations of [OI] and o$\mathrm{H}_{2} \mathrm{O}$ at $63 \mu \mathrm{m}$ in YSOs, including Class 0, I, II, transitional discs, and debris discs, for a total of 432 observations of 362 sources.

We note that the $[\mathrm{OI}]$ emission line intensity, as well as detection fractions, decreases during the evolution from Class 0 to debris discs. However, we did not see a difference in [OI] emission between Class 0 and Class I, nor between Class II and transition discs. o- $\mathrm{H}_{2} \mathrm{O}$ emission line intensity also decreases from Class 0 and I to more evolved sources (Class II and transition discs).

By means of comparing the fluxes computed from the central spaxel, the central $3 \times 3$ spaxels and the integrated IFU, we detected extended emission in the [OI] line for a total of 77 sources. For those sources showing hints of extended [OI] emission, we obtained line emission maps and residual maps, and confirmed residual emission in 71 sources. The fraction of sources showing extended emission decreases dramatically from Class 0 , where $63 \%$ of the sources show extended emission, to Class II, where only $17 \%$ of the sources show extended emission.

We detected extended $\mathrm{o}-\mathrm{H}_{2} \mathrm{O}$ line emission in only one source.

For 30 sources in the sample we were able to fit multiple components to the line emission profile, which is indicative of different contributions to the line (envelope, discs, winds, and jets).

We have tested previously identified correlations in the entire sample. The [OI] line emission correlates with continuum emission at $63 \mu \mathrm{m}$ for all classes, with the exception of of debris discs.

We confirm the correlation between $[\mathrm{OI}]$ and $\mathrm{o}-\mathrm{H}_{2} \mathrm{O}$ at $63 \mu \mathrm{m}$, and tentatively see a change in slope in the correlation between class 0 and I sources and class II sources.

We have identified new correlations with continuum emission between 4.6 and $22 \mu \mathrm{m}$, indicating an extended emitting region (from the inner disc to tens of au) as the origin of the disc contribution.

Acknowledgements. The authors would like to thank the anonymous referee for a very fruitful discussion that helped to improve the quality of the paper. P.R.M. acknowledges funding from the ESA Research Fellowship program. C.E. and B.M. are partly supported by Spanish Grant AYA 2014-55840-P.

\section{References}

Alcalá, J. M., Natta, A., Manara, C. F., et al. 2014, A\&A, 561, A2

Andre, P., Ward-Thompson, D., \& Barsony, M. 1993, ApJ, 406, 122

Bouvier, J., \& Appenzeller, I. 1992, A\&AS, 92, 481

Bouy, H., \& Martín, E. L. 2009, A\&A, 504, 981

Carpenter, J. M., Mamajek, E. E., Hillenbrand, L. A., \& Meyer, M. R. 2006, ApJ, 651, L49

Comerón, F. 2008, in The Lupus Clouds, Handbook of Star Forming Regions, ASP, ed. B. Reipurth, 295

de la Reza, R., Torres, C. A. O., Quast, G., Castilho, B. V., \& Vieira, G. L. 1989, ApJ, 343, L61

de Zeeuw, P. T., Hoogerwerf, R., de Bruijne, J. H. J., Brown, A. G. A., \& Blaauw, A. 1999, AJ, 117, 354

Dent, W. R. F., Thi, W. F., Kamp, I., et al. 2013, PASP, 125, 477

Donaldson, J. K., Roberge, A., Chen, C. H., et al. 2012, ApJ, 753, 147

Dzib, S., Loinard, L., Mioduszewski, A. J., et al. 2010, ApJ, 718, 610

Erickson, K. L., Wilking, B. A., Meyer, M. R., Robinson, J. G., \& Stephenson, L. N. 2011, AJ, 142, 140

Fedele, D., Bruderer, S., van Dishoeck, E. F., et al. 2013, A\&A, 559, A77

Feigelson, E. D., \& Babu, J. G. 2012, Modern Statistical Methods for Astronomy (Cambridge, UK: Cambridge University Press)

Furlan, E., Watson, D. M., McClure, M. K., et al. 2009, ApJ, 703, 1964

Gorti, U., \& Hollenbach, D. 2008, ApJ, 683, 287

Gorti, U., Hollenbach, D., Najita, J., \& Pascucci, I. 2011, ApJ, 735, 90

Gray, R. O., Corbally, C. J., Garrison, R. F., et al. 2006, AJ, 132, 161

Green, J. D., Evans, II, N. J., Jørgensen, J. K., et al. 2013, ApJ, 770, 123

Gregorio-Hetem, J., Lepine, J. R. D., Quast, G. R., Torres, C. A. O., \& de La Reza, R. 1992, AJ, 103, 549

Hales, A. S., De Gregorio-Monsalvo, I., Montesinos, B., et al. 2014, AJ, 148, 47

Harlan, E. A. 1974, AJ, 79, 682

Herczeg, G. J., Karska, A., Bruderer, S., et al. 2012, A\&A, 540, A84

Hirota, T., Bushimata, T., Choi, Y. K., et al. 2008, PASJ, 60, 37

Houk, N. 1978, Michigan Catalogue of Two-dimensional Spectral Types for the HD Stars (University of Michigan Press)

Houk, N. 1982, Michigan Catalogue of Two-dimensional Spectral Types for the HD Stars, Volume_3. Declinations -40_deg0 to -26_deg0 (University of Michigan Press)

Houk, N., \& Cowley, A. P. 1975, Michigan Catalogue of Two-dimensional Spectral Types for the HD Stars, Vol. I. Declinations -90_ to -53 deg0 (University of Michigan Press)

Houk, N., \& Smith-Moore, M. 1988, Michigan Catalogue of Two-dimensional Spectral Types for the HD Stars, Vol. 4, Declinations -26deg0 to -12deg0 (University of Michigan Press) 
P. Riviere-Marichalar et al.: Herschel-PACS observations of far-IR lines in young stellar objects. I.

Houk, N., \& Swift, C. 1999, in Michigan Spectral Survey (Univ. Michigan Press), 5, 0

Howard, C. D., Sandell, G., Vacca, W. D., et al. 2013, ApJ, 776, 21

Hughes, J., Hartigan, P., Krautter, J., \& Kelemen, J. 1994, AJ, 108, 1071

Kamp, I., Tilling, I., Woitke, P., Thi, W.-F., \& Hogerheijde, M. 2010, A\&A, 510, A18

Kamp, I., Woitke, P., Pinte, C., et al. 2011, A\&A, 532, A85

Karska, A., Herczeg, G. J., van Dishoeck, E. F., et al. 2013, A\&A, 552, A141

Karska, A., Herpin, F., Bruderer, S., et al. 2014a, A\&A, 562, A45

Karska, A., Kristensen, L. E., van Dishoeck, E. F., et al. 2014b, A\&A, 572, A9

Keane, J. T., Pascucci, I., Espaillat, C., et al. 2014, ApJ, 787, 153

Kenyon, S. J., Gómez, M., \& Whitney, B. A. 2008, in Low Mass Star Formation in the Taurus-Auriga Clouds, Handbook of Star Forming Regions, ASP, ed B. Reipurth, 405

Köhler, R., Kunkel, M., Leinert, C., \& Zinnecker, H. 2000, A\&A, 356, 541

Kraemer, K. E., Jackson, J. M., \& Lane, A. P. 1998, ApJ, 503, 785

Kraus, A. L., Ireland, M. J., Martinache, F., \& Lloyd, J. P. 2008, ApJ, 679, 762

Lada, C. J. 1987, in Star Forming Regions, eds. M. Peimbert, \& J. Jugaku, IAU Symp., 115, 1

Lada, C. J., \& Wilking, B. A. 1984, ApJ, 287, 610

Lebreton, J., Augereau, J.-C., Thi, W.-F., et al. 2012, A\&A, 539, A17

Lee, J.-E., Lee, J., Lee, S., Evans, II, N. J., \& Green, J. D. 2014, ApJS, 214, 21

Levenhagen, R. S., \& Leister, N. V. 2006, MNRAS, 371, 252

Lindberg, J. E., Jørgensen, J. K., Green, J. D., et al. 2014, A\&A, 565, A29

López Martí, B., Jiménez-Esteban, F., \& Solano, E. 2011, A\&A, 529, A108

Luhman, K. L. 2004, ApJ, 602, 816

Luhman, K. L. 2007, ApJS, 173, 104

Luhman, K. L. 2008, in Chamaeleon, Handbook of Star Forming Regions, ASP, ed. B. Reipurth, 169

Luhman, K. L., Allen, P. R., Espaillat, C., Hartmann, L., \& Calvet, N. 2010, ApJS, 186, 111

Mamajek, E. E., Lawson, W. A., \& Feigelson, E. D. 1999, ApJ, 516, L77

Manoj, P., Bhatt, H. C., Maheswar, G., \& Muneer, S. 2006, ApJ, 653, 657

Marsh, K. A., Silverstone, M. D., Becklin, E. E., et al. 2002, ApJ, 573, 425

Martin, E. L., Montmerle, T., Gregorio-Hetem, J., \& Casanova, S. 1998, MNRAS, 300, 733

Mathews, G. S., Dent, W. R. F., Williams, J. P., et al. 2010, A\&A, 518, L127

Mathews, G. S., Pinte, C., Duchêne, G., Williams, J. P., \& Ménard, F. 2013, A\&A, 558, A66

McClure, M. K., Furlan, E., Manoj, P., et al. 2010, ApJS, 188, 75

Meeus, G., Pinte, C., Woitke, P., et al. 2010, A\&A, 518, L124

Meeus, G., Salyk, C., Bruderer, S., et al. 2013, A\&A, 559, A84

Melnick, G. J., Tolls, V., Neufeld, D. A., et al. 2008, ApJ, 683, 876

Mendigutía, I., Calvet, N., Montesinos, B., et al. 2011, A\&A, 535, A99

Mohanty, S., Greaves, J., Mortlock, D., et al. 2013, ApJ, 773, 168

Mora, A., Merín, B., Solano, E., et al. 2001, A\&A, 378, 116
Mortier, A., Oliveira, I., \& van Dishoeck, E. F. 2011, MNRAS, 418, 1194 Neuhäuser, R., \& Forbrich, J. 2008, in The Corona Australis Star Forming Region, Handbook of Star Forming Regions, ASP, ed. B. Reipurth, 735 Nilsson, R., Liseau, R., Brandeker, A., et al. 2009, A\&A, 508, 1057

Nisini, B., Santangelo, G., Antoniucci, S., et al. 2013, A\&A, 549, A16 Nisini, B., Santangelo, G., Giannini, T., et al. 2015, ApJ, 801, 121 Pilbratt, G. L., Riedinger, J. R., Passvogel, T., et al. 2010, A\&A, 518, L1

Podio, L., Kamp, I., Flower, D., et al. 2012, A\&A, 545, A44

Poglitsch, A., Waelkens, C., Geis, N., et al. 2010, A\&A, 518, L2

Prato, L., Greene, T. P., \& Simon, M. 2003, ApJ, 584, 853

Preibisch, T., Guenther, E., Zinnecker, H., et al. 1998, A\&A, 333, 619

Preibisch, T., Brown, A. G. A., Bridges, T., Guenther, E., \& Zinnecker, H. 2002, AJ, 124, 404

Ricci, L., Testi, L., Natta, A., \& Brooks, K. J. 2010, A\&A, 521, A66

Riviere-Marichalar, P., Barrado, D., Augereau, J.-C., et al. 2012a, A\&A, 546, L8

Riviere-Marichalar, P., Ménard, F., Thi, W. F., et al. 2012b, A\&A, 538, L3

Riviere-Marichalar, P., Pinte, C., Barrado, D., et al. 2013, A\&A, 555, A67

Riviere-Marichalar, P., Barrado, D., Montesinos, B., et al. 2014, A\&A, 565, A68

Riviere-Marichalar, P., Bayo, A., Kamp, I., et al. 2015, A\&A, 575, A19

Rydgren, A. E. 1980, AJ, 85, 444

Spezzi, L., Alcalá, J. M., Covino, E., et al. 2008, ApJ, 680, 1295

Strom, K. M., Strom, S. E., Edwards, S., Cabrit, S., \& Skrutskie, M. F. 1989, AJ, 97, 1451

Sturm, B., Bouwman, J., Henning, T., et al. 2010, A\&A, 518, L129

Thi, W.-F., Mathews, G., Ménard, F., et al. 2010, A\&A, 518, L125

Thi, W. F., Ménard, F., Meeus, G., et al. 2013, A\&A, 557, A111

Thi, W.-F., Pinte, C., Pantin, E., et al. 2014, A\&A, 561, A50

Tilling, I., Woitke, P., Meeus, G., et al. 2012, A\&A, 538, A20

Torres, C. A. O., Quast, G. R., da Silva, L., et al. 2006, A\&A, 460, 695

Vacca, W. D., \& Sandell, G. 2011, ApJ, 732, 8

van Dishoeck, E. F., Kristensen, L. E., Benz, A. O., et al. 2011, PASP, 123, 138

van Kempen, T. A., Green, J. D., Evans, N. J., et al. 2010a, A\&A, 518, L128

van Kempen, T. A., Kristensen, L. E., Herczeg, G. J., et al. 2010b, A\&A, 518, L121

Vieira, S. L. A., Corradi, W. J. B., Alencar, S. H. P., et al. 2003, AJ, 126, 2971

Webb, R. A., Zuckerman, B., Platais, I., et al. 1999, ApJ, 512, L63

Weinberger, A. J., Becklin, E. E., Zuckerman, B., \& Song, I. 2004, AJ, 127, 2246

Wichmann, R., Krautter, J., Covino, E., et al. 1997, A\&A, 320, 185

Wilking, B. A., Gagné, M., \& Allen, L. E. 2008, in Star Formation in the $\rho$ Ophiuchi Molecular Cloud, Handbook of Star Forming Regions, ASP, ed. B. Reipurth, 351

Woitke, P., Pinte, C., Tilling, I., et al. 2010, MNRAS, 405, L26

Woitke, P., Riaz, B., Duchêne, G., et al. 2011, A\&A, 534, A44

Wright, E. L., Eisenhardt, P. R. M., Mainzer, A. K., et al. 2010, AJ, 140, 1868

Zuckerman, B., \& Song, I. 2004a, ApJ, 603, 738

Zuckerman, B., \& Song, I. 2004b, ARA\&A, 42, 685 
Appendix A: Line emission maps
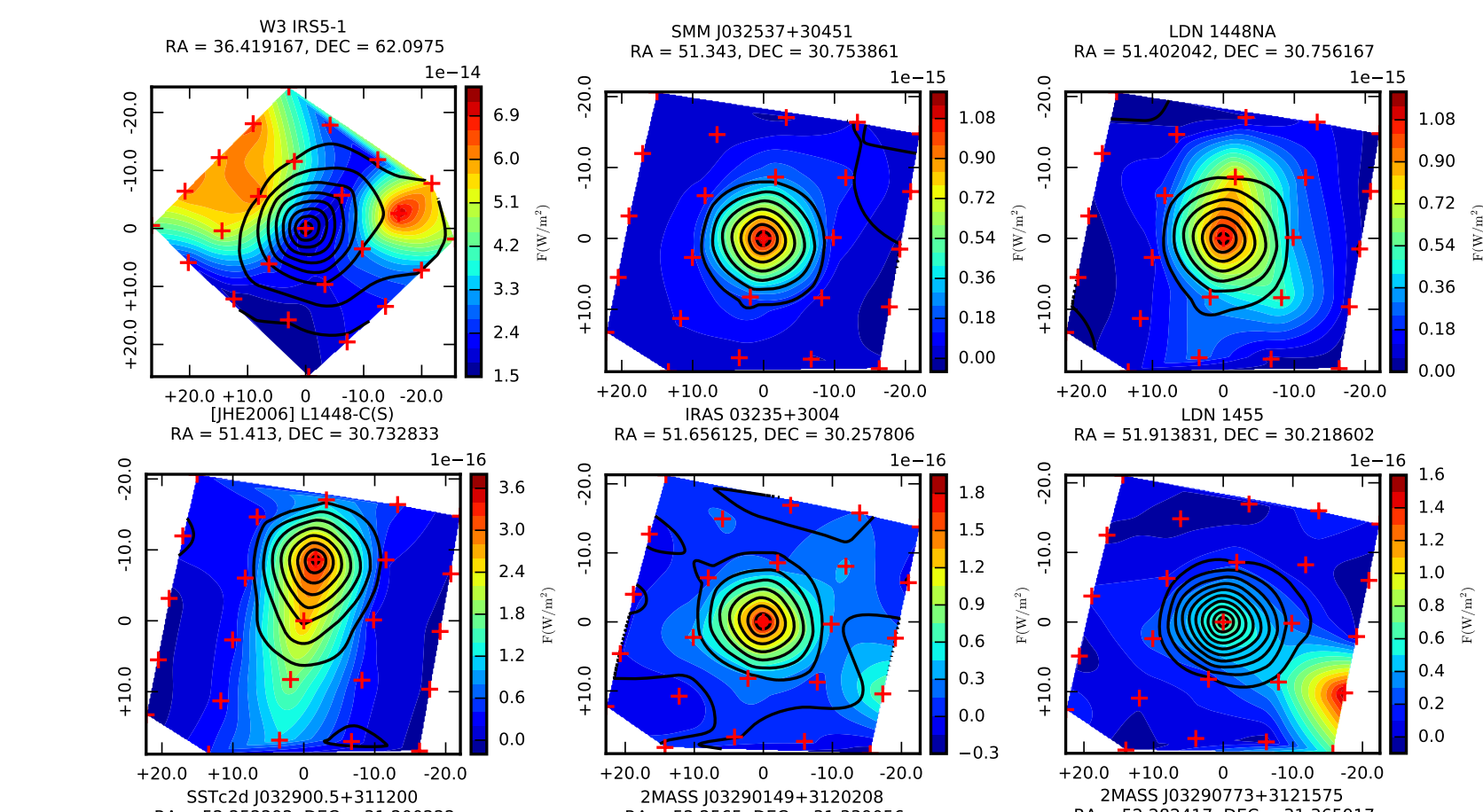

RA $=51.913831, \mathrm{DEC}=30.218602$
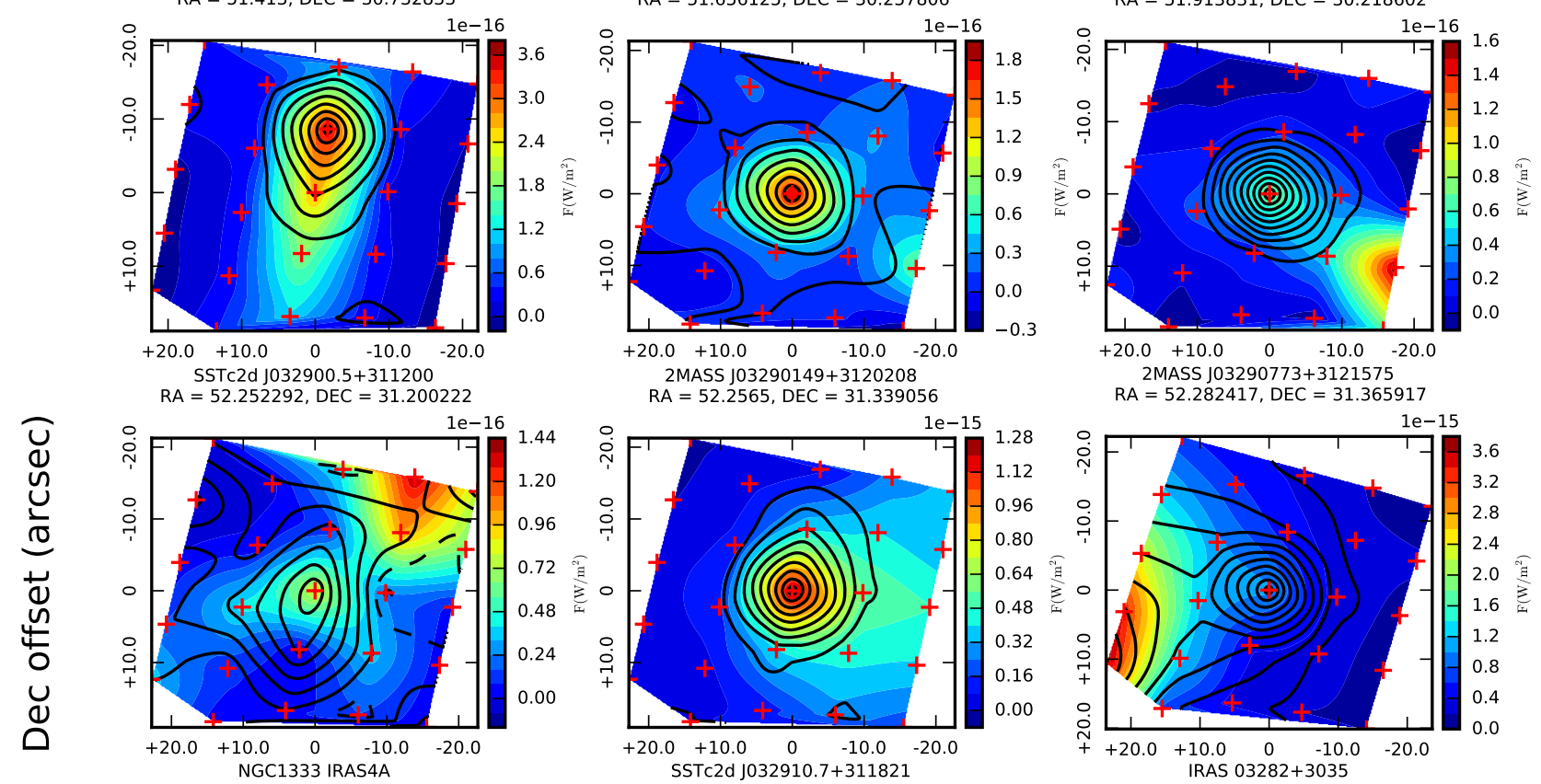

$\mathrm{RA}=52.2565, \mathrm{DEC}=31.339056$
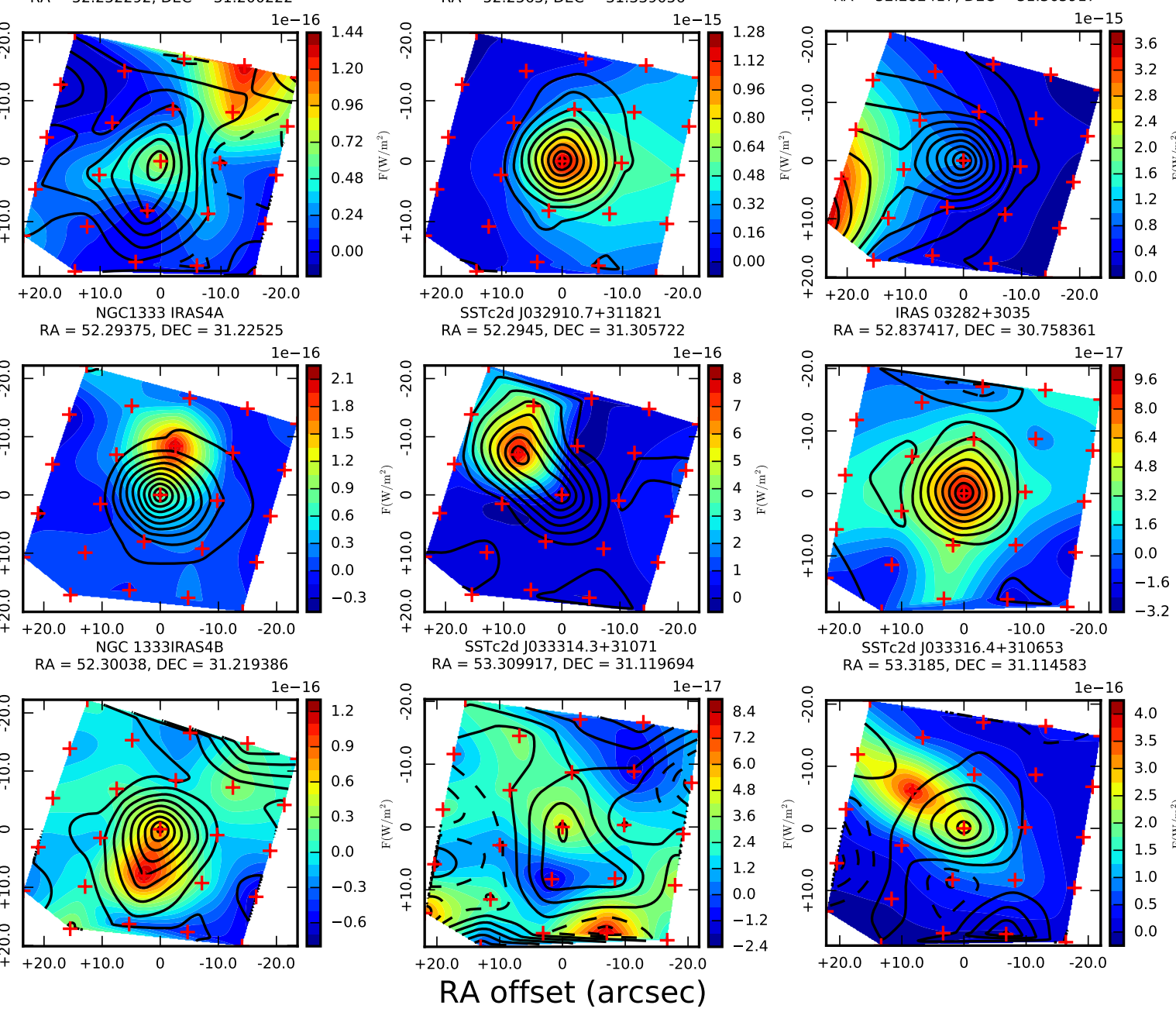

Fig. A.1. [OI] line emission at $63 \mu \mathrm{m}$ (coloured contours) and $63 \mu \mathrm{m}$ continuum contours (solid black lines) for sources identified as extended by any of the three tests used. The positions of spaxels are marked with red plus signs. 
P. Riviere-Marichalar et al.: Herschel-PACS observations of far-IR lines in young stellar objects. I.

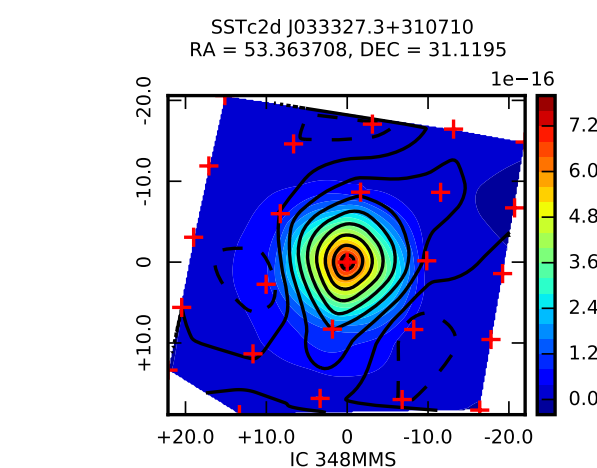

$\mathrm{RA}=55.986833, \mathrm{DEC}=32.051306$

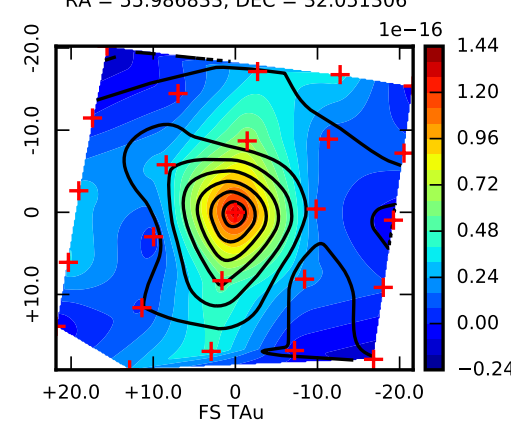

$\mathrm{RA}=65.507737, \mathrm{DEC}=26.958584$

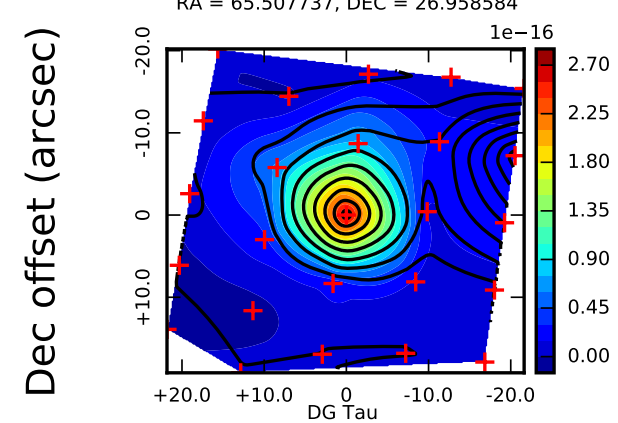

$\mathrm{RA}=66.767929, \mathrm{DEC}=26.104629$

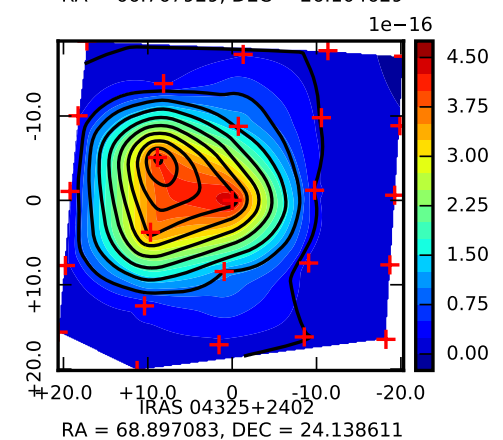

$\mathrm{RA}=68.897083, \mathrm{DEC}=24.138611$

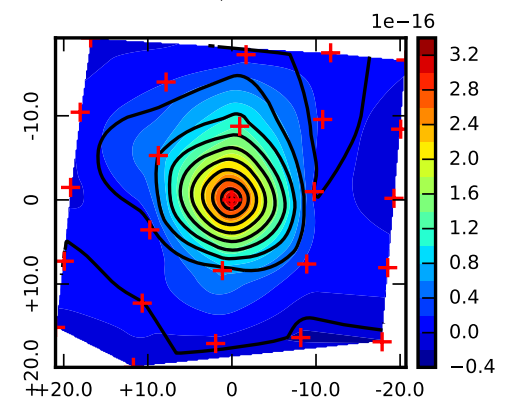

$\begin{aligned} \mathrm{B} 1 \mathrm{a} \\ \mathrm{RA}\end{aligned}=53.320177, \mathrm{DEC}=31.133035$

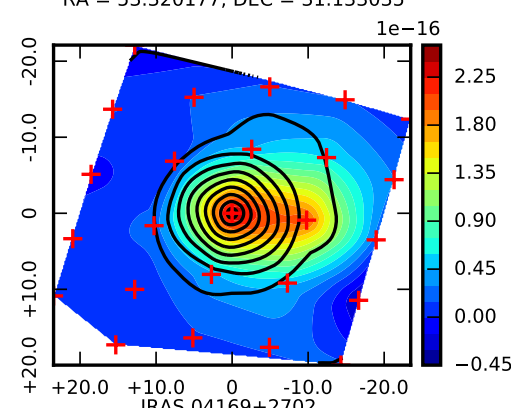

$\mathrm{RA}=64.993333, \mathrm{DEC}=27.165833$

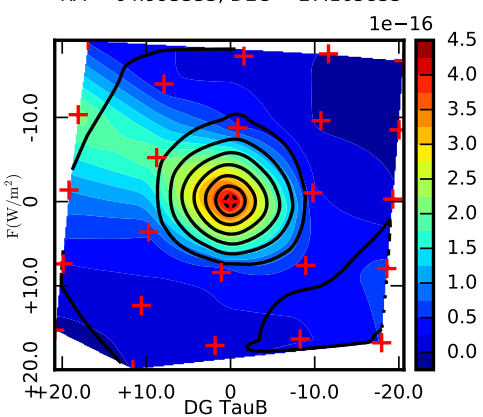

$R A=66.758852, D E C=26.092219$

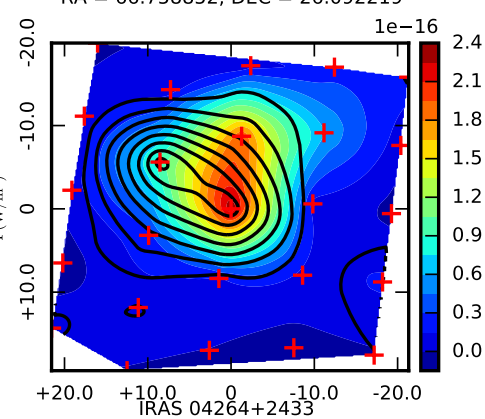

$\mathrm{RA}=67.375, \mathrm{DEC}=24.665278$

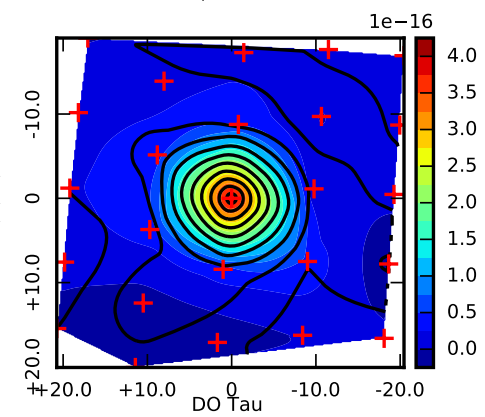

$\mathrm{RA}=69.617184, \mathrm{DEC}=26.181248$

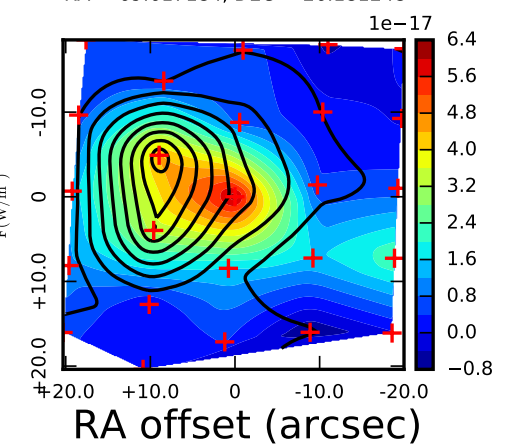

IRAS $03407+3152$

$\mathrm{RA}=55.9855, \mathrm{DEC}=32.014667$

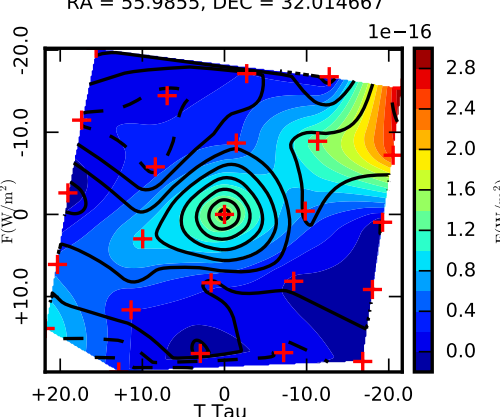

$\mathrm{RA}=65.49699, \mathrm{DEC}=19.535786$

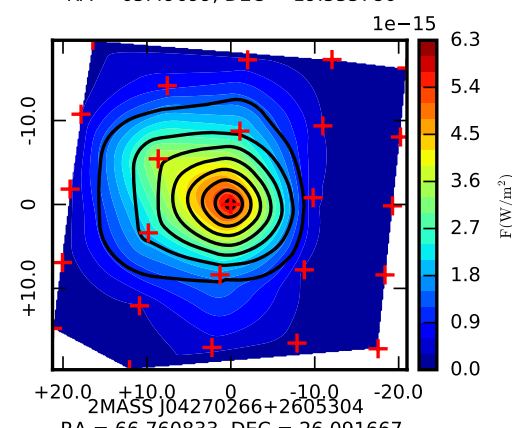

$\mathrm{RA}=66.760833, \mathrm{DEC}=26.091667$

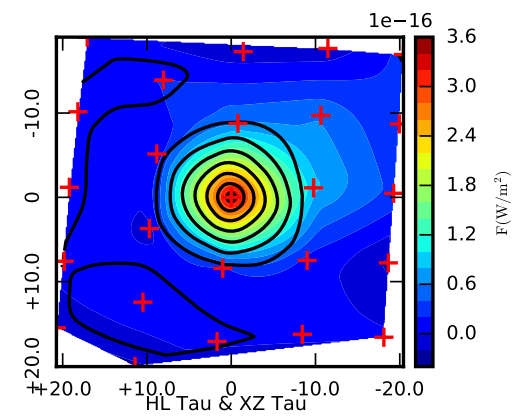

$\mathrm{RA}=67.913581, \mathrm{DEC}=18.232737$

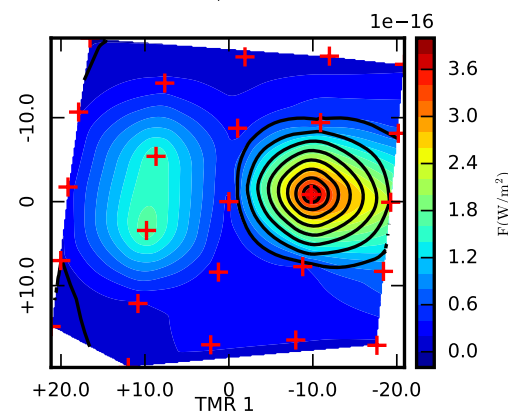

$\mathrm{RA}=69.807083, \mathrm{DEC}=25.889167$

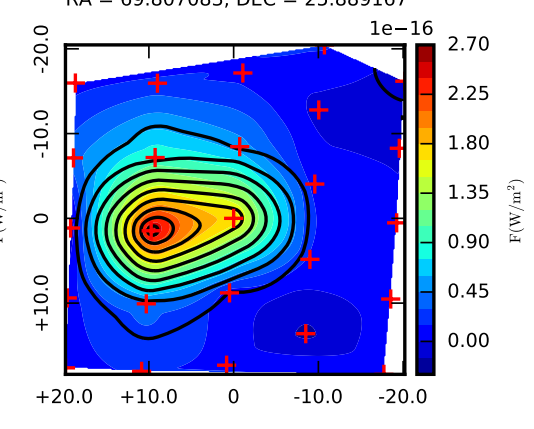

Fig. A.1. continued. 
TMC 1A

$\mathrm{RA}=69.894211, \mathrm{DEC}=25.696854$

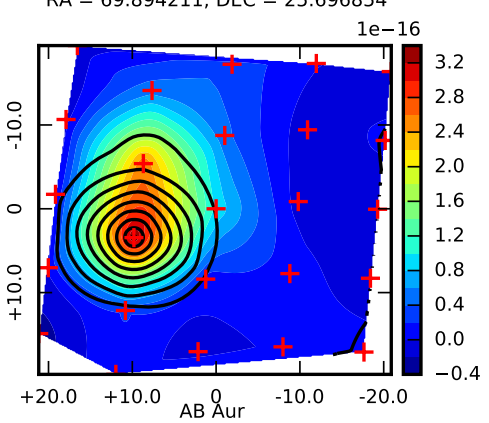

$\mathrm{RA}=73.939858, \mathrm{DEC}=30.551888$

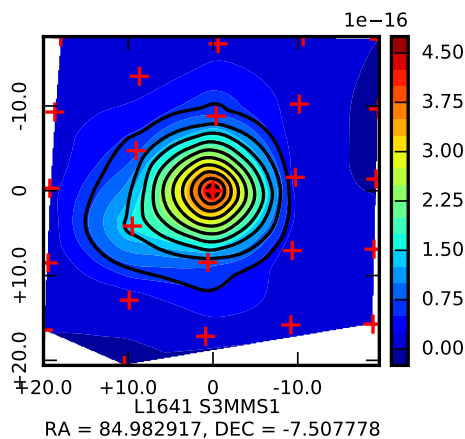

$\mathrm{RA}=84.982917, \mathrm{DEC}=-7.507778$

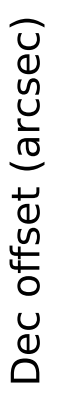

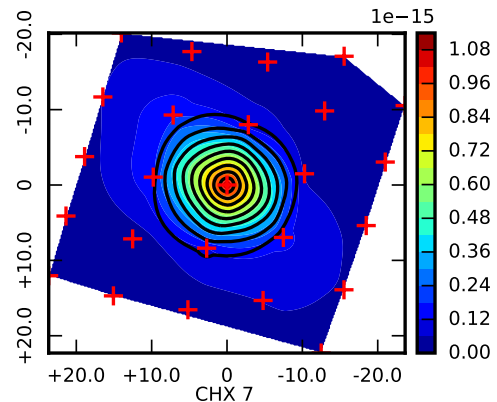

$\mathrm{RA}=166.564356, \mathrm{DEC}=-77.365974$

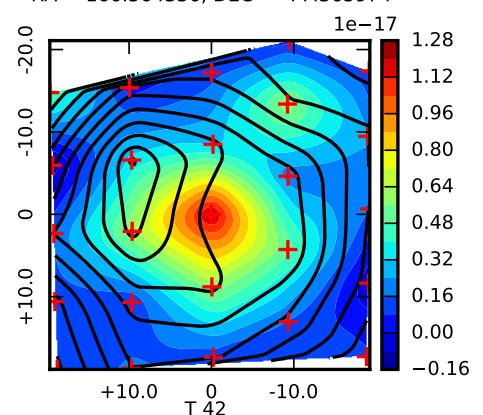

$\mathrm{RA}=167.47374, \mathrm{DEC}=-76.573678$

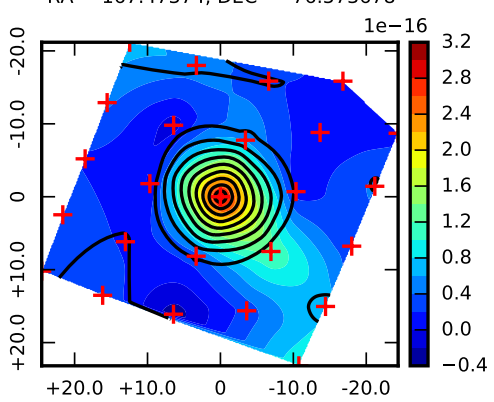

L 1527

$R A=69.974583, D E C=26.052722$

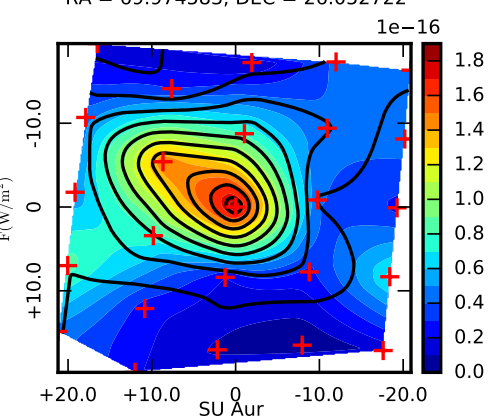

$\mathrm{RA}=73.99584, \mathrm{DEC}=30.567759$

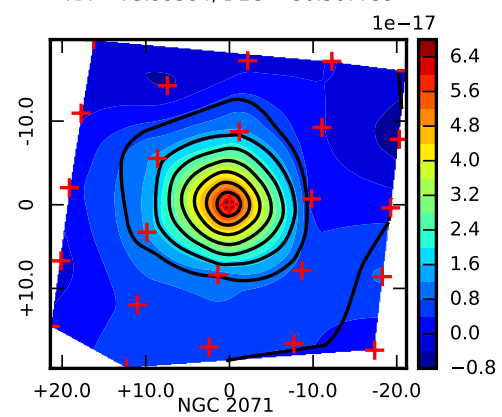

$\mathrm{RA}=86.768333, \mathrm{DEC}=0.363611$

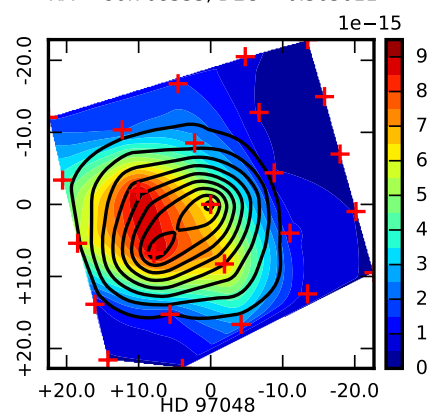

$\mathrm{RA}=167.011313, \mathrm{DEC}=-77.654789$

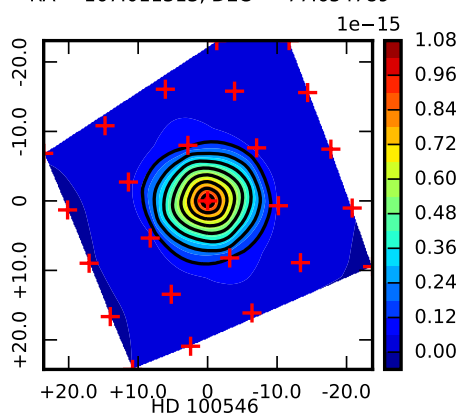

$\mathrm{RA}=173.355511, \mathrm{DEC}=-70.194857$

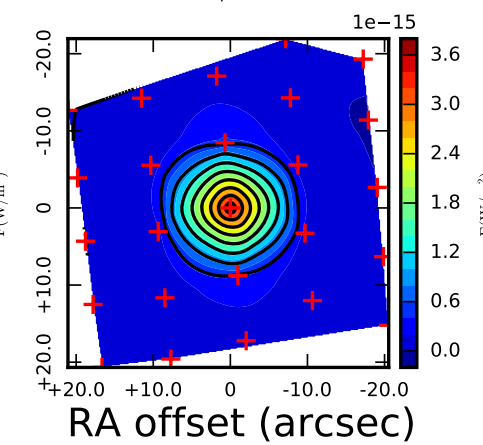

TMC 1

$\mathrm{RA}=70.301667, \mathrm{DEC}=25.776667$

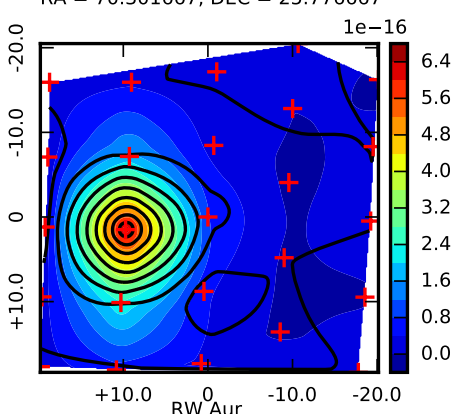

$\mathrm{RA}=76.956215, \mathrm{DEC}=30.4021$

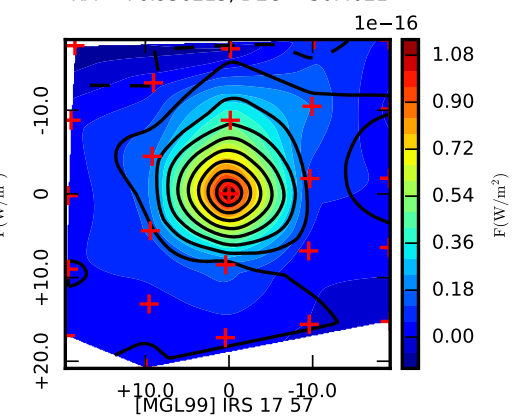

$\mathrm{RA}=131.644711, \mathrm{DEC}=-43.908012$

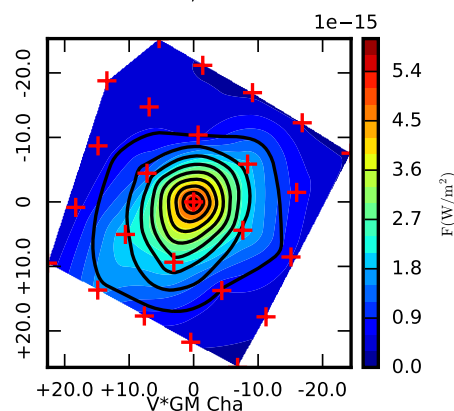

$\mathrm{RA}=167.368792, \mathrm{DEC}=-76.557889$

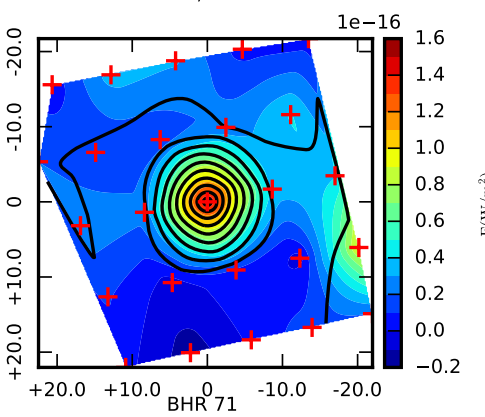

$\mathrm{RA}=180.40125, \mathrm{DEC}=-65.148056$

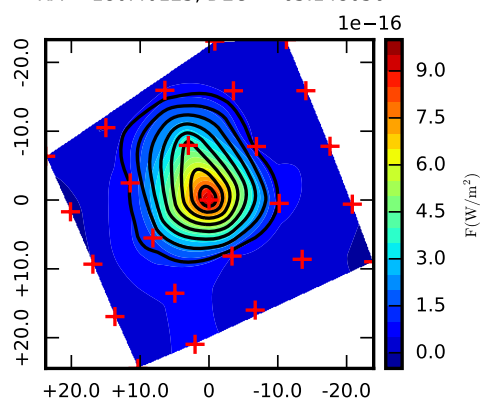

Fig. A.1. continued. 
P. Riviere-Marichalar et al.: Herschel-PACS observations of far-IR lines in young stellar objects. I.

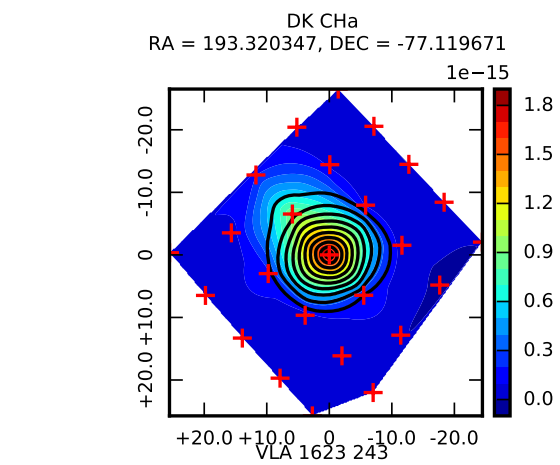

$\mathrm{RA}=246.609532, \mathrm{DEC}=-24.408152$

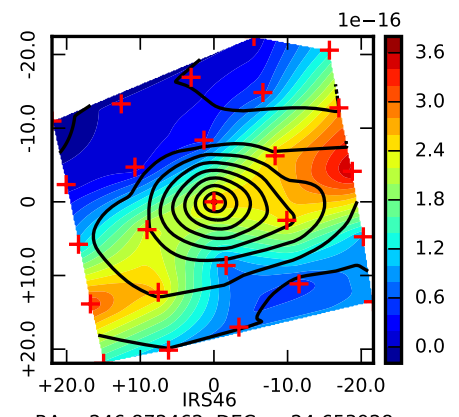

$\mathrm{RA}=246.872462, \mathrm{DEC}=-24.653928$
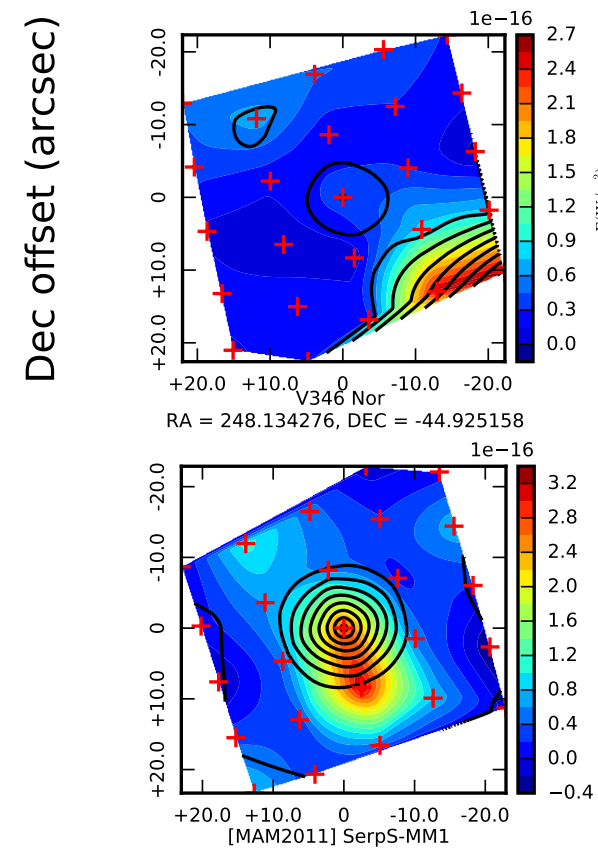

$\mathrm{RA}=277.407083, \mathrm{DEC}=-1.849389$

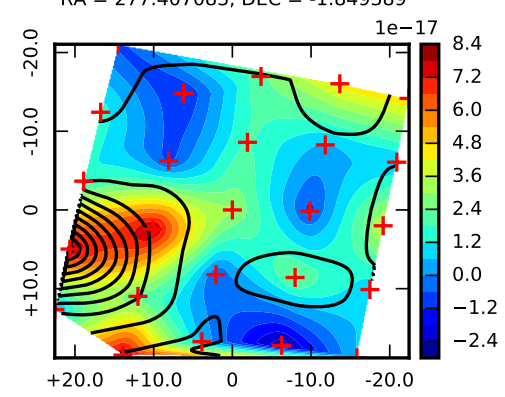

DoAr 21
RA $=246.512616$, DEC $=-24.393425$

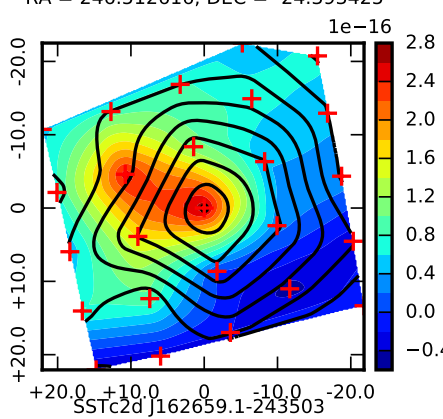

$\mathrm{RA}=246.746282, \mathrm{DEC}=-24.584137$

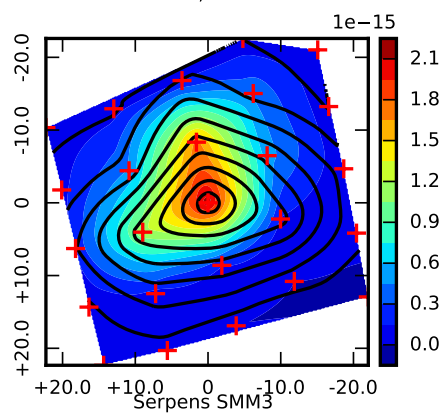

$\mathrm{RA}=277.49904, \mathrm{DEC}=1.232656$

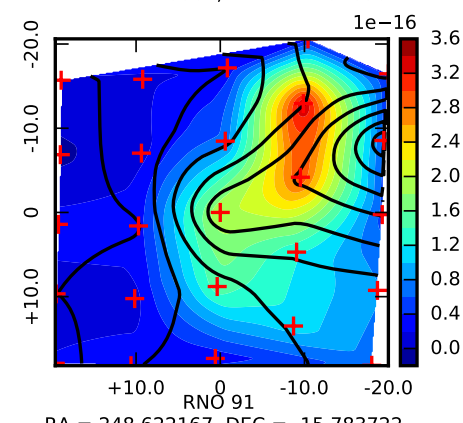

$\mathrm{RA}=248.622167, \mathrm{DEC}=-15.783722$

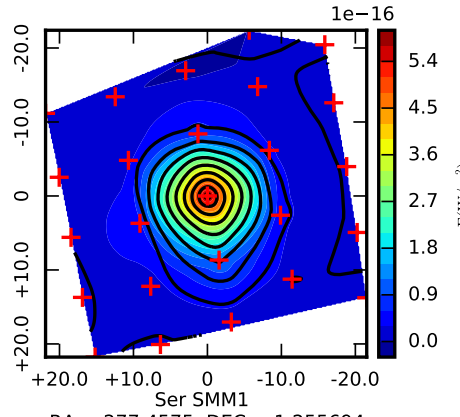

$\mathrm{RA}=277.4575, \mathrm{DEC}=1.255694$

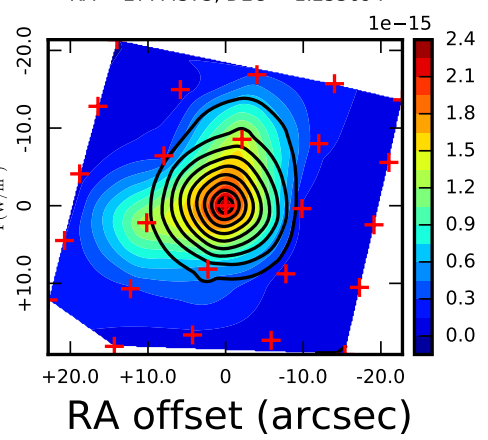

GSS 30 IRS 1

$\mathrm{RA}=246.588868, \mathrm{DEC}=-24.384372$

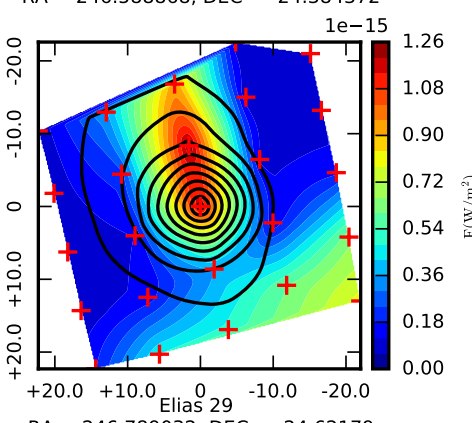

$\mathrm{RA}=246.789032, \mathrm{DEC}=-24.62179$

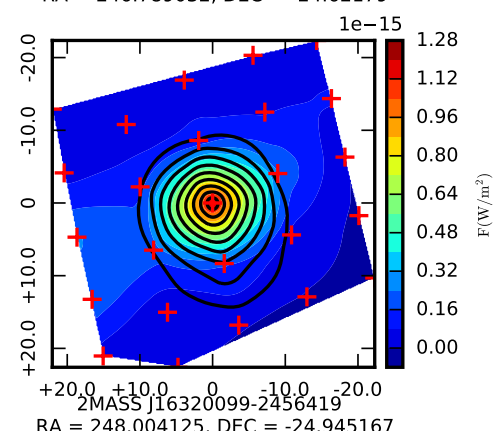

$\mathrm{RA}=248.004125, \mathrm{DEC}=-24.945167$

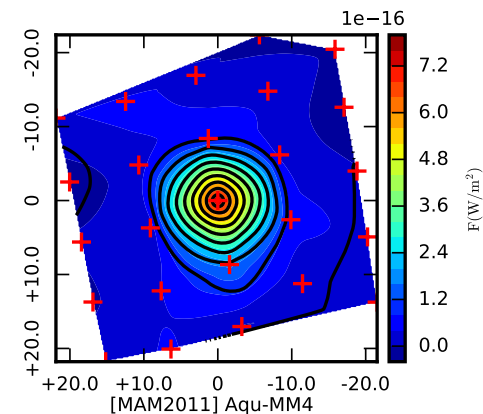

$\mathrm{RA}=277.285833, \mathrm{DEC}=-1.511889$

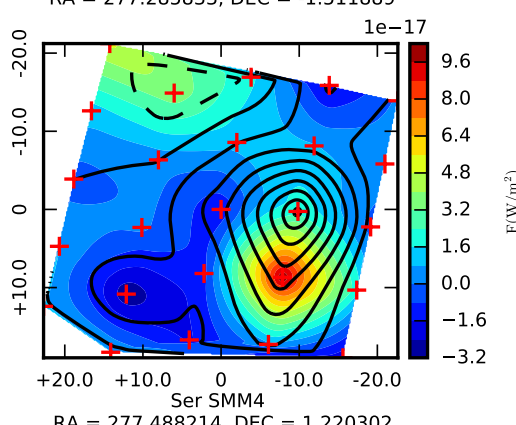

$\mathrm{RA}=277.488214, \mathrm{DEC}=1.220302$

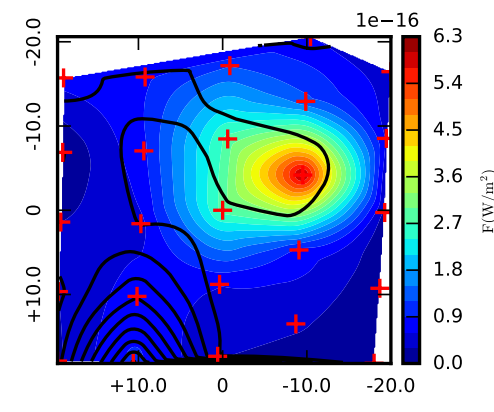

Fig. A.1. continued. 

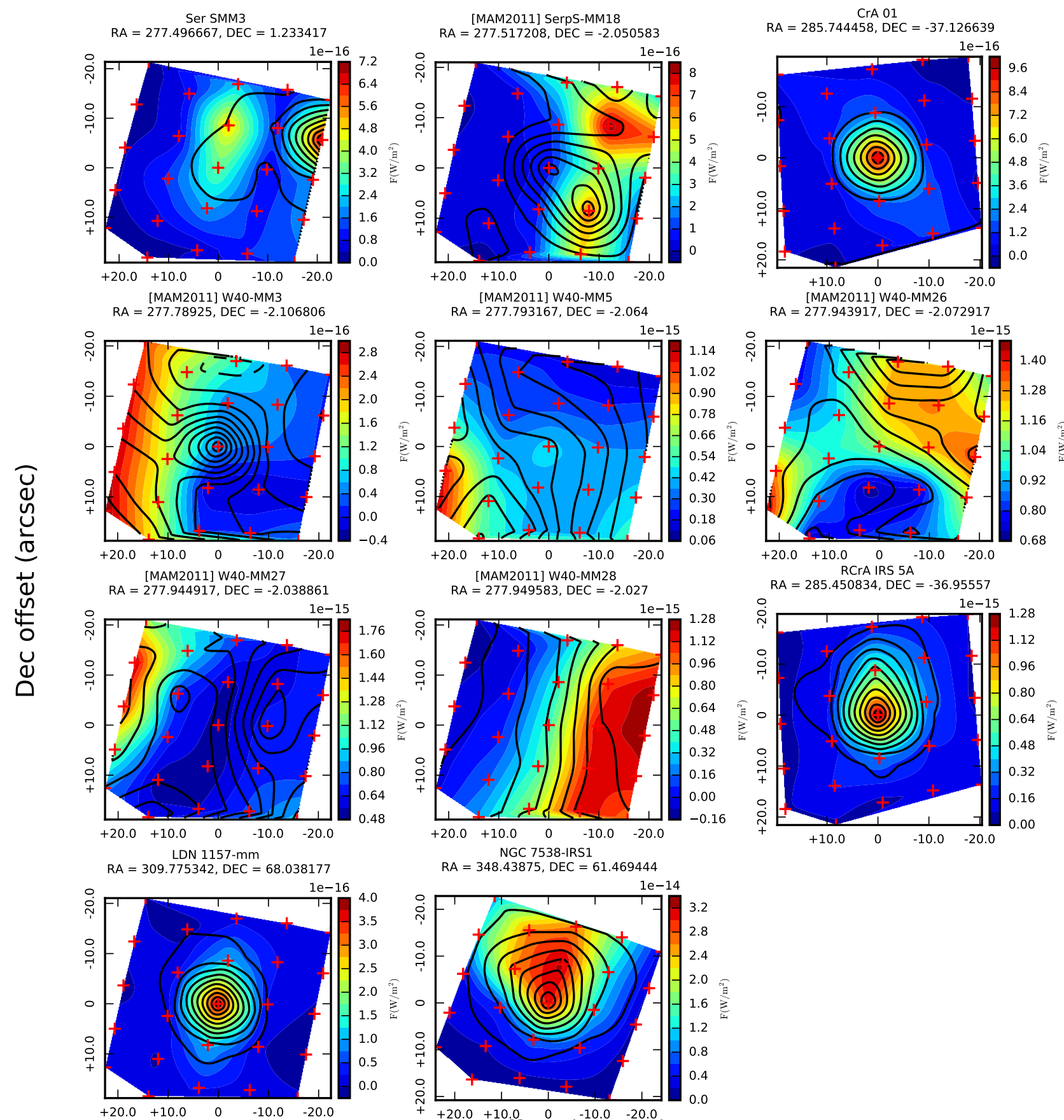

$\mathrm{RA}=348.43875, \mathrm{DEC}=61.469444$

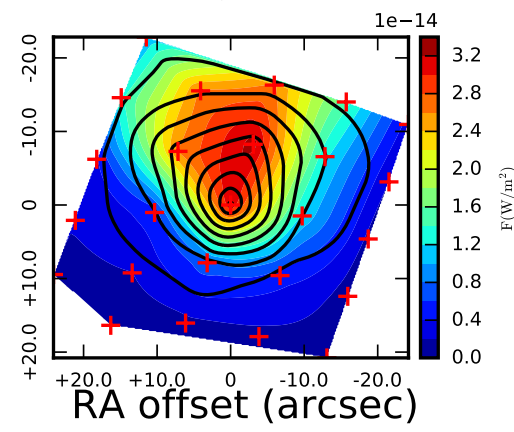

Fig. A.1. continued. 
P. Riviere-Marichalar et al.: Herschel-PACS observations of far-IR lines in young stellar objects. I.

\section{Appendix B: Residual line emission maps}
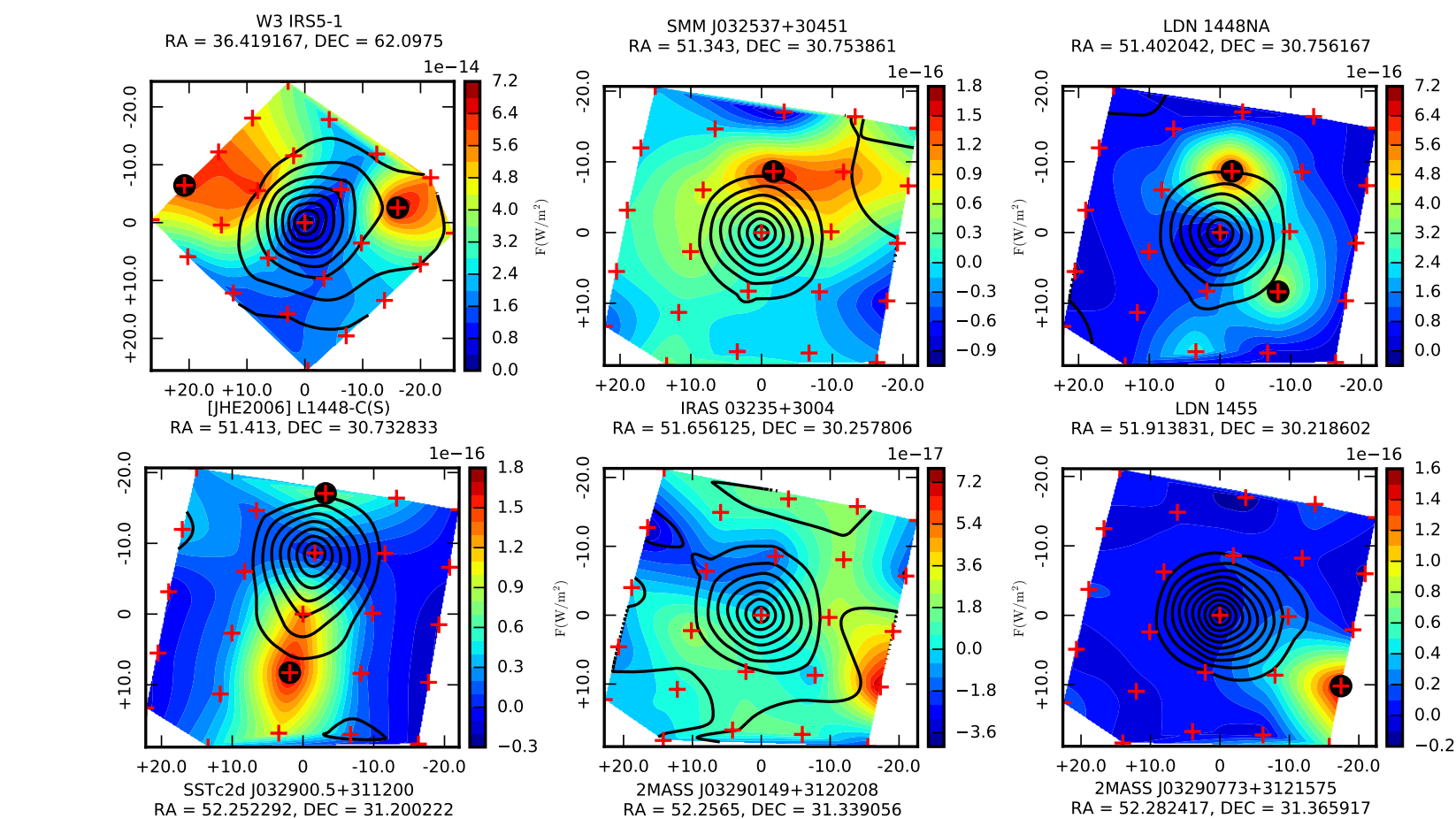

RA $=51.913831$, DEC $=30.218602$
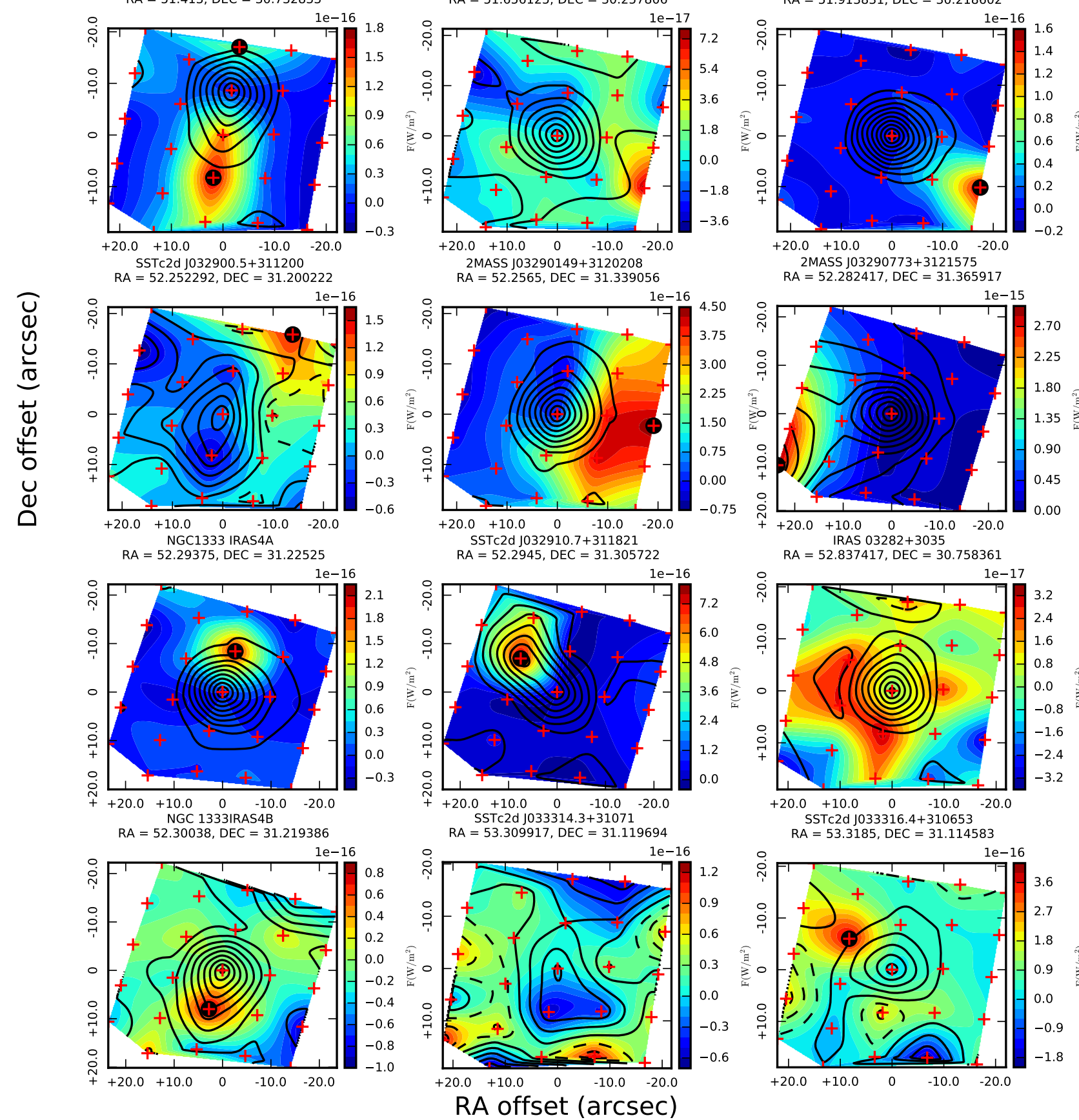

Fig. B.1. [OI] residual line emission at $63 \mu \mathrm{m}$ (coloured contours) and $63 \mu \mathrm{m}$ continuum contours (solid black lines) for sources identified as extended by any of the three tests used. The positions of spaxels are marked with red plus signs. Spaxel with $5 \sigma$ residual detections are surrounded by black dots. 


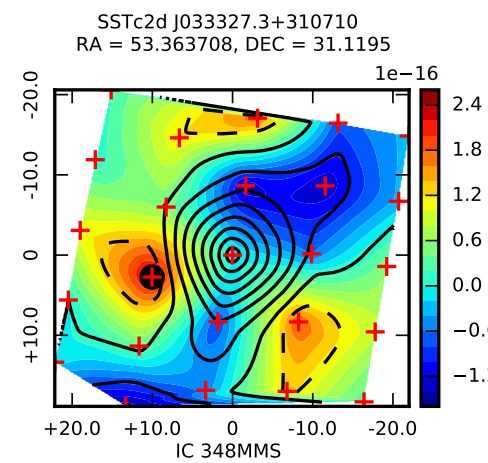

$R A=55.986833, D E C=32.051306$

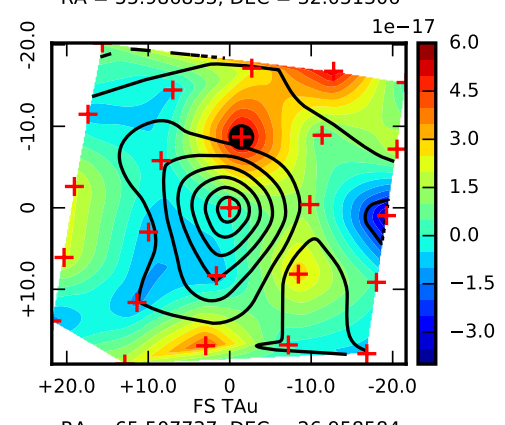

$R A=65.507737, D E C=26.958584$

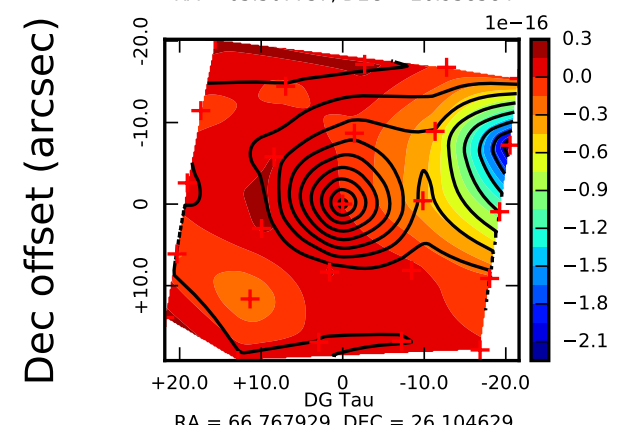

$\mathrm{RA}=66.767929, \mathrm{DEC}=26.104629$
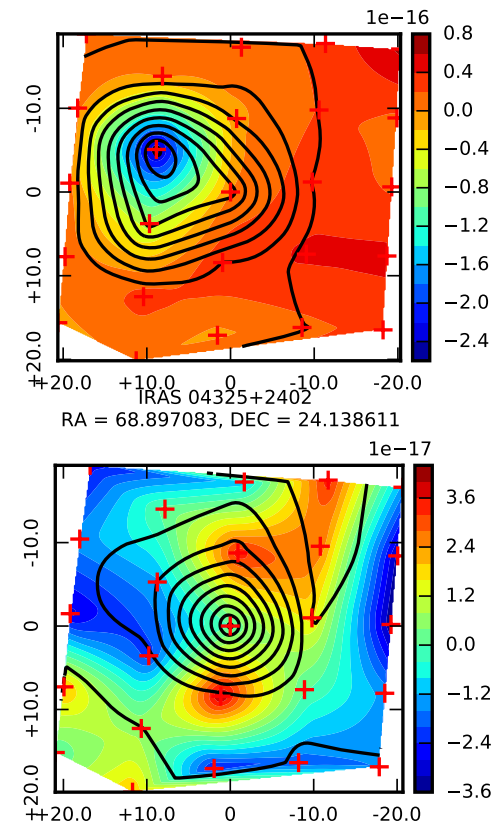

B1 a

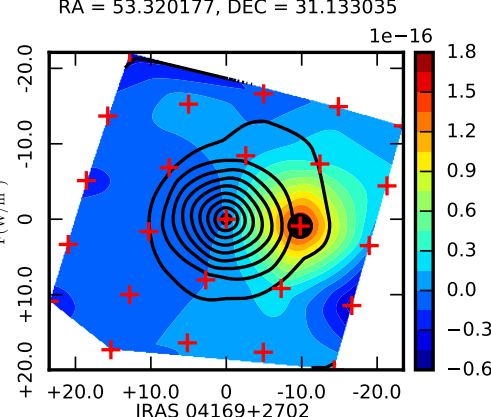

$\mathrm{RA}=64.993333, \mathrm{DEC}=27.165833$

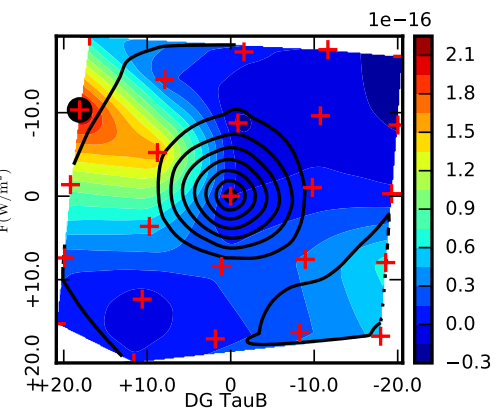

$R A=66.758852, D E C=26.092219$

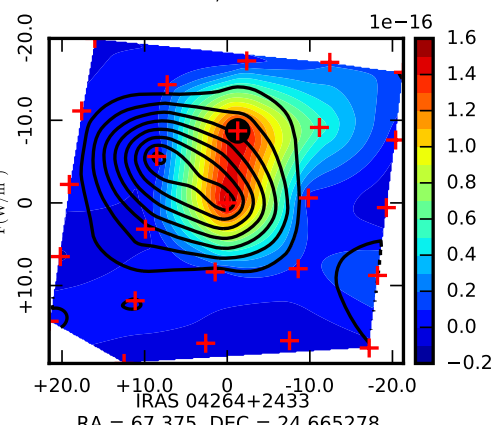

$\mathrm{RA}=67.375, \mathrm{DEC}=24.665278$
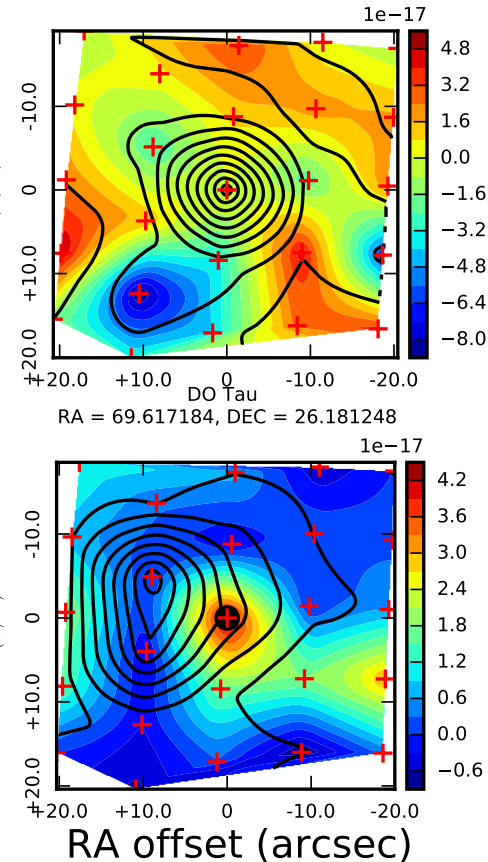

IRAS $03407+3152$

$\mathrm{RA}=55.9855, \mathrm{DEC}=32.014667$

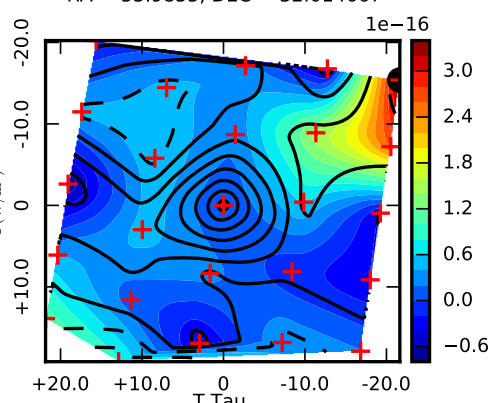

$\mathrm{RA}=65.49699, \mathrm{DEC}=19.535786$
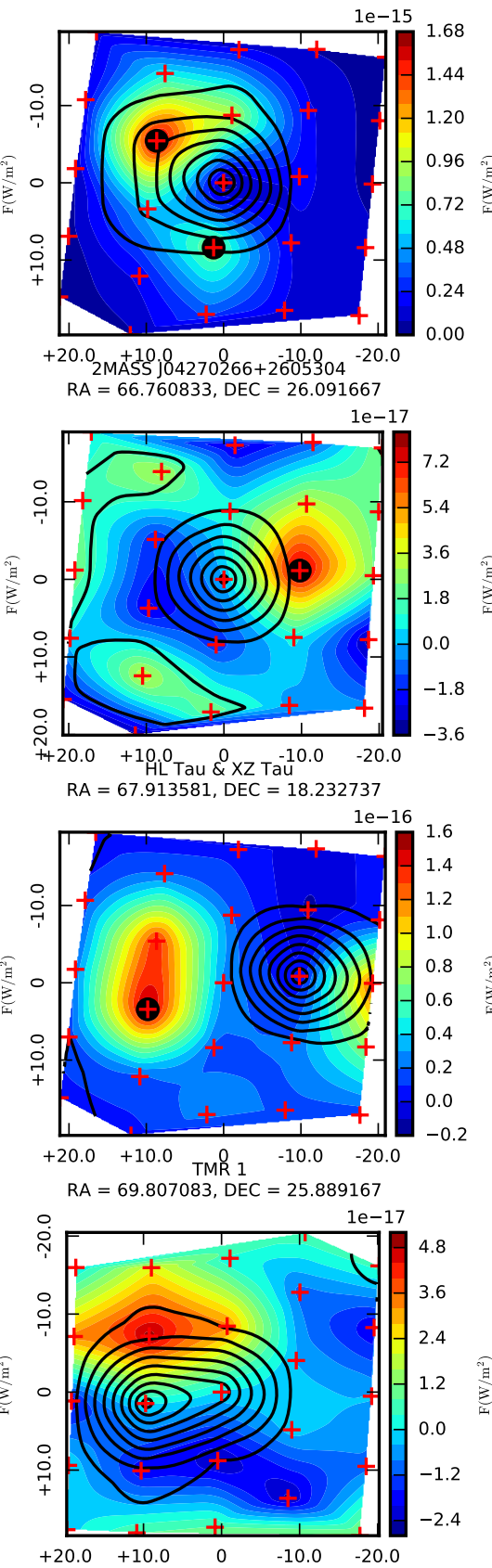

Fig. B.1. continued. 
P. Riviere-Marichalar et al.: Herschel-PACS observations of far-IR lines in young stellar objects. I.
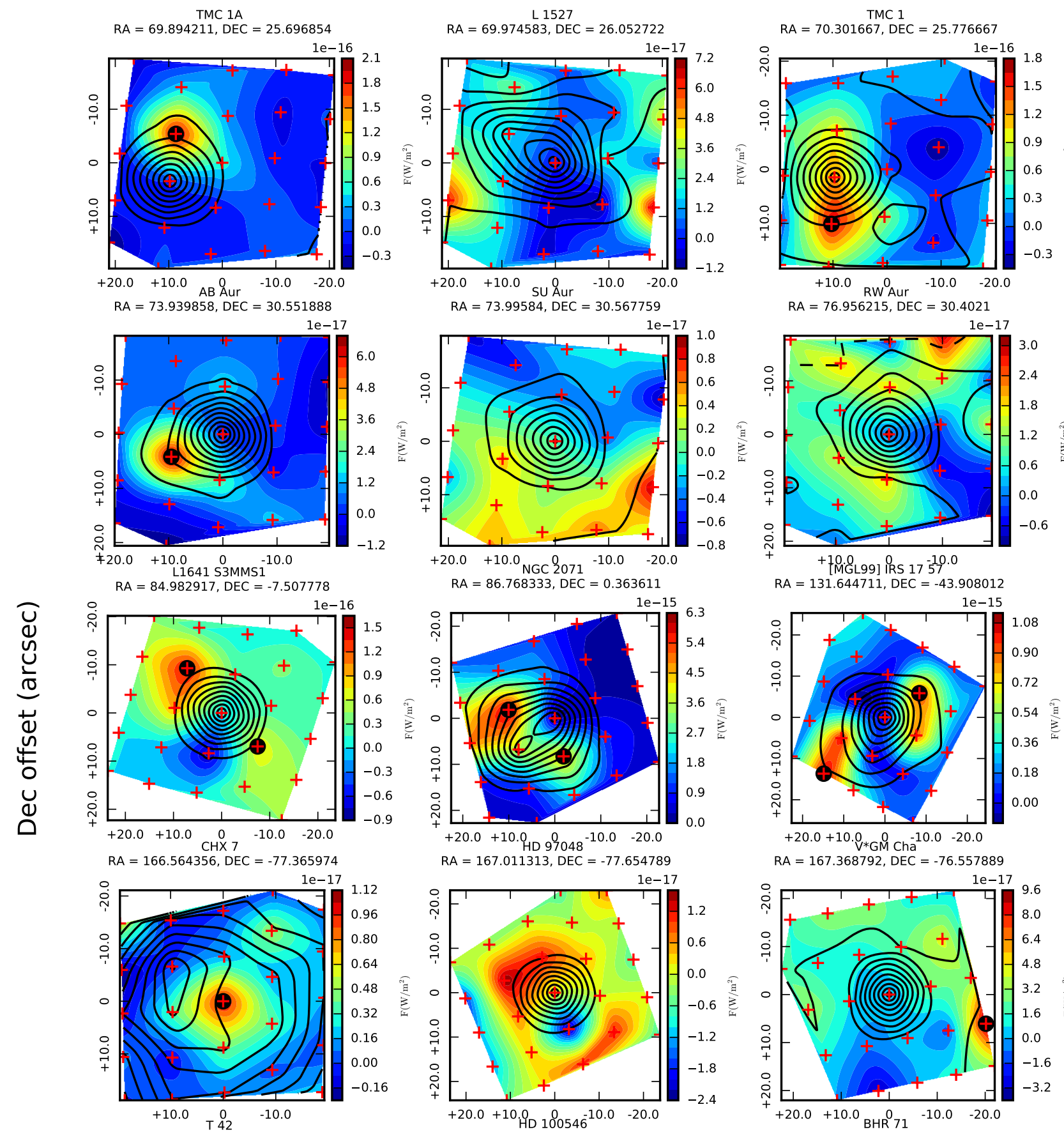

$R A=167.47374, D E C=-76.573678$
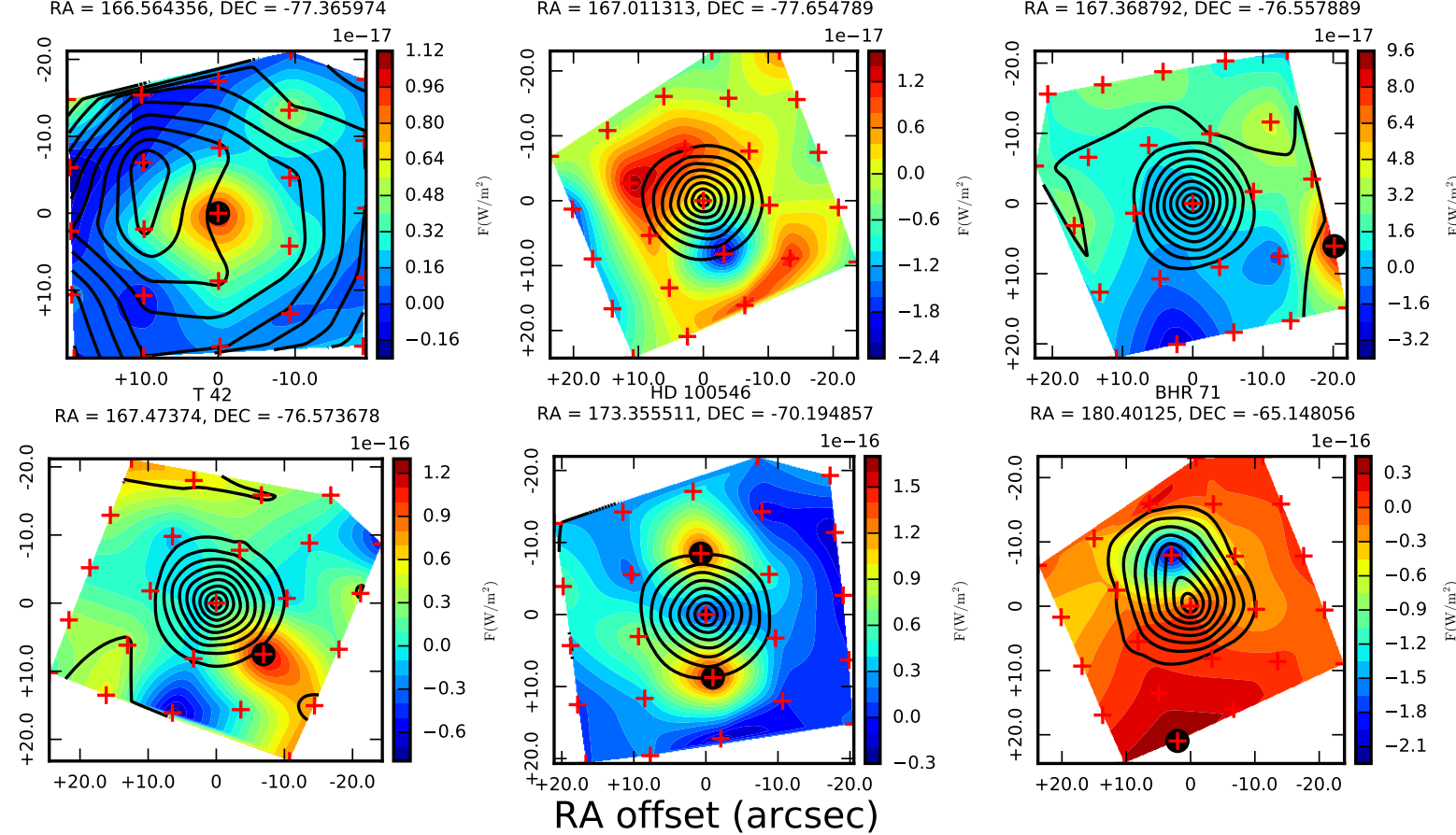

Fig. B.1. continued. 


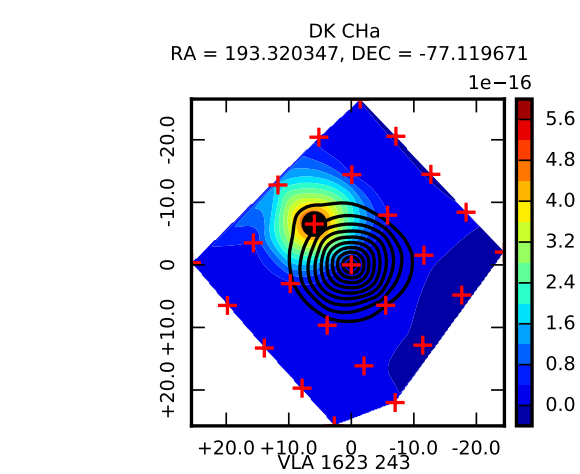

$\mathrm{RA}=246.609532, \mathrm{DEC}=-24.408152$

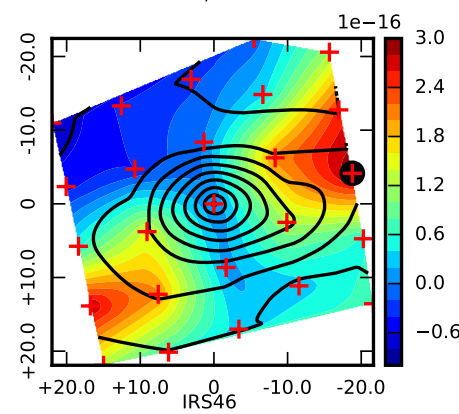

$R A=246.872462, D E C=-24.653928$

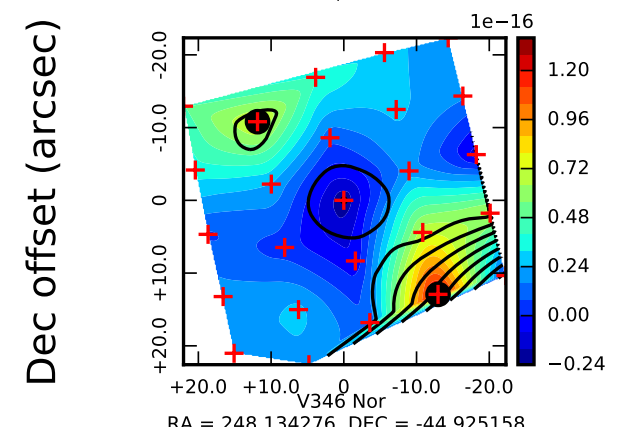

$\mathrm{RA}=248.134276, \mathrm{DEC}=-44.925158$

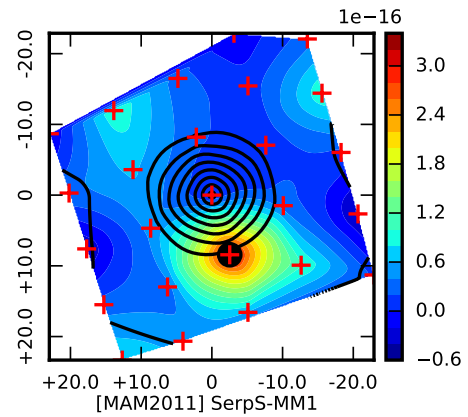

$\mathrm{RA}=277.407083, \mathrm{DEC}=-1.849389$

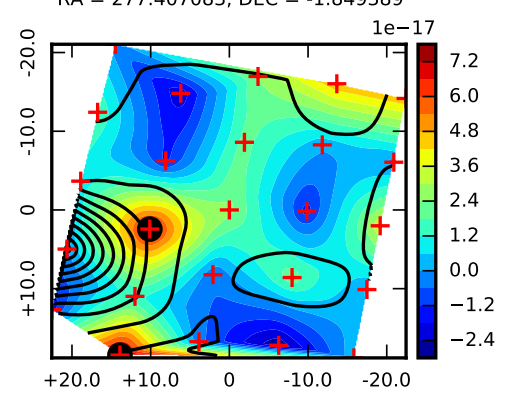

DoAr 21
RA $=246.512616$, DEC $=-24.393425$

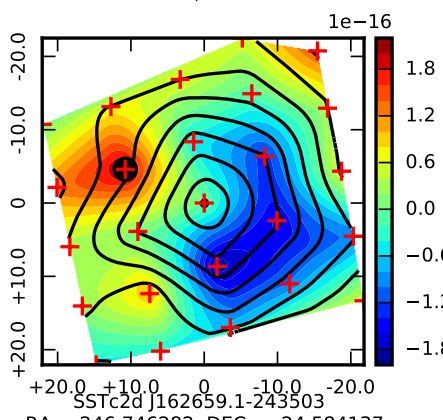

$\mathrm{RA}=246.746282, \mathrm{DEC}=-24.584137$

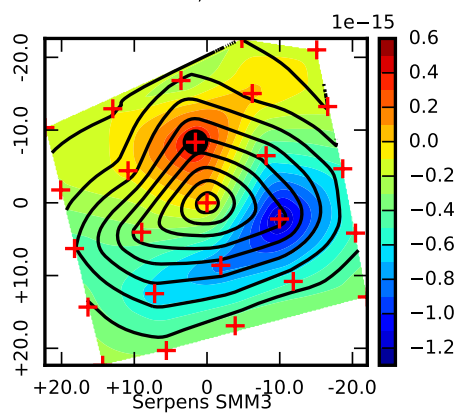

$\mathrm{RA}=277.49904, \mathrm{DEC}=1.232656$

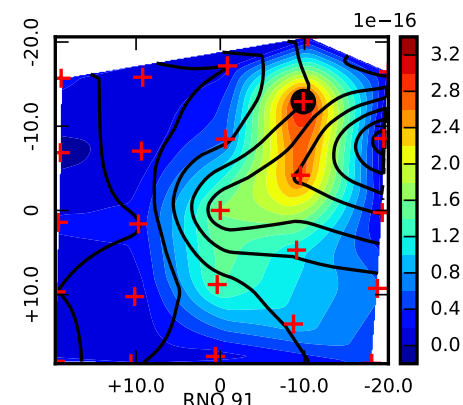

$R A=248.622167, D E C=-15.783722$

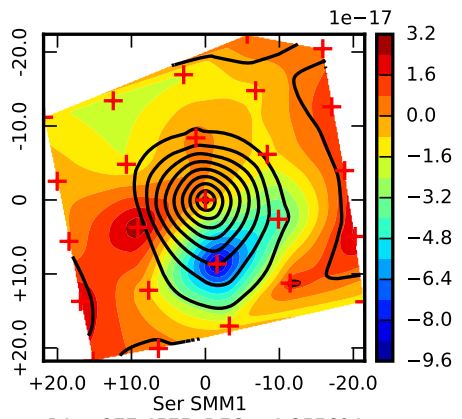

$\mathrm{RA}=277.4575, \mathrm{DEC}=1.255694$

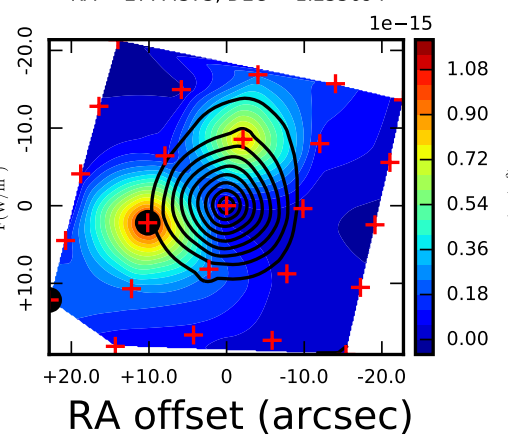

GSS 30 IRS 1

$R A=246.588868, D E C=-24.384372$

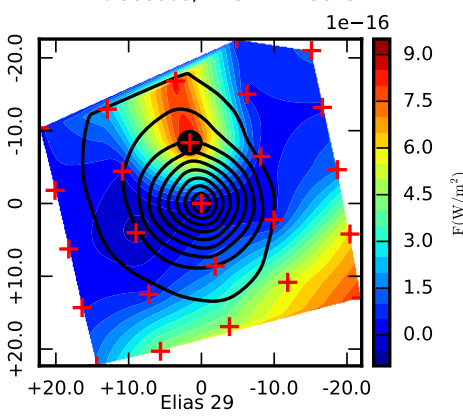

$\mathrm{RA}=246.789032, \mathrm{DEC}=-24.62179$

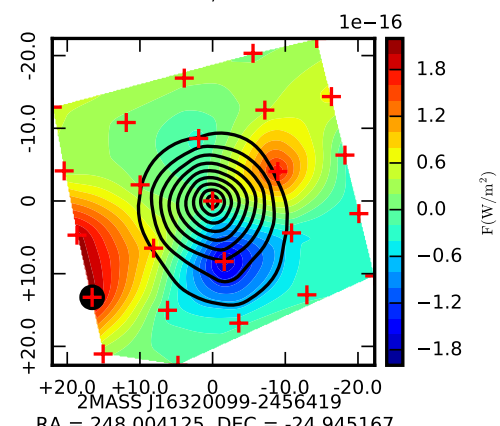

$\mathrm{RA}=248.004125, \mathrm{DEC}=-24.945167$

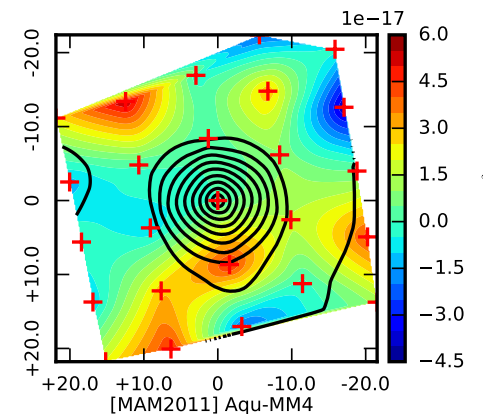

$R A=277.285833, D E C=-1.511889$

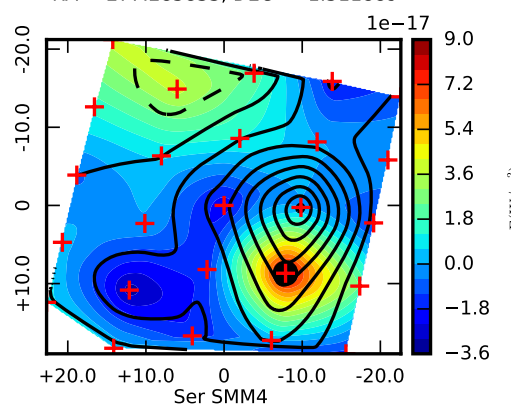

$\mathrm{RA}=277.488214, \mathrm{DEC}=1.220302$

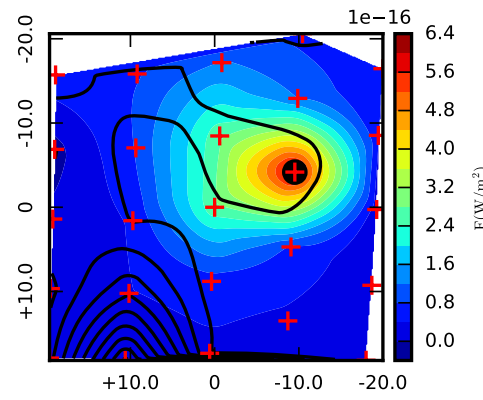

Fig. B.1. continued. 
P. Riviere-Marichalar et al.: Herschel-PACS observations of far-IR lines in young stellar objects. I.
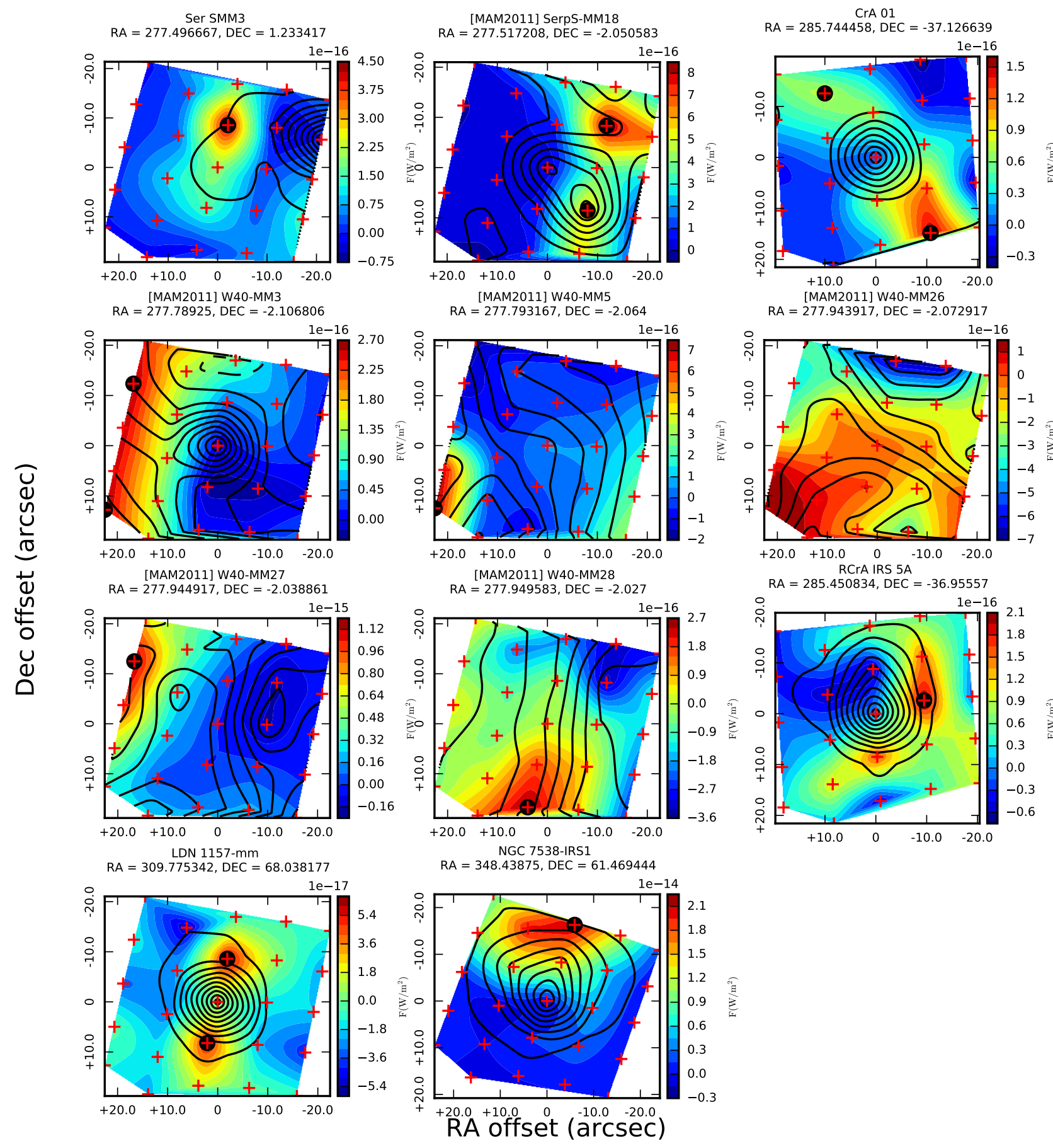

Fig. B.1. continued. 